\title{
NEWTON'S METHOD BACKPROPAGATION FOR COMPLEX-VALUED HOLOMORPHIC MULTILAYER PERCEPTRONS
}

\author{
DIANA THOMSON LA CORTE AND YI MING ZOU
}

\begin{abstract}
The study of Newton's method in complex-valued neural networks faces many difficulties. In this paper, we derive Newton's method backpropagation algorithms for complex-valued holomorphic multilayer perceptrons, and investigate the convergence of the one-step Newton steplength algorithm for the minimization of real-valued complex functions via Newton's method. To provide experimental support for the use of holomorphic activation functions, we perform a comparison of using sigmoidal functions versus their Taylor polynomial approximations as activation functions by using the algorithms developed in this paper and the known gradient descent backpropagation algorithm. Our experiments indicate that the Newton's method based algorithms, combined with the use of polynomial activation functions, provide significant improvement in the number of training iterations required over the existing algorithms.
\end{abstract}

\section{INTRODUCTION}

The use of fully complex-valued neural networks to solve real-valued as well as complex-valued problems in physical applications has become increasingly popular in the neural network community in recent years [1, 2, 3. Complex-valued neural networks pose unique problems, however. Consider the problem of choosing the activation functions for a neural network. Real-valued activation functions for real-valued neural networks are commonly taken to be everywhere differentiable and bounded. Typical activation functions used for real-valued neural networks are the sigmoidal, hyperbolic tangent, and hyperbolic secant functions

$$
f(x)=\frac{1}{1+\exp (-x)}, \tanh (x)=\frac{e^{x}-e^{-x}}{e^{x}+e^{-x}}, \text { and } \operatorname{sech}(x)=\frac{2}{e^{x}+e^{-x}} .
$$


For activation functions of complex-valued networks, an obvious choice is to use the complex counterparts of these real-valued functions. However, as complex-valued functions, these functions are no longer differentiable and bounded near 0 , since they have poles near 0 . Different approaches have been proposed in the literature to address this problem.

Liouville's theorem tells us that there is no non-constant complexvalued function which is both bounded and differentiable on the whole complex plane [4]. On the basis of Liouville's theorem, [5] asserts that an entire function is not suitable as an activation function for a complex-valued neural network and claims boundedness as an essential property of the activation function. Some authors followed this same reasoning and use the so-called "split" functions of the type $f(z)=$ $f(x+i y)=f_{1}(x)+i f_{2}(y)$ where $f_{1}, f_{2}$ are real-valued functions, typically taken to be one of the sigmoidal functions [6, 7, 8]. Such activation functions have the advantage of easily modeling data with symmetry about the real and imaginary axes. However, this yields complexvalued neural networks which are close to real-valued networks of double dimensions and are not fully complex-valued [1. Amplitude-phasetype activation functions have the type $f(z)=f_{3}(|z|) \exp (i \arg (z))$ where $f_{3}$ is a real-valued function. These process wave information well, but have the disadvantage of preserving phase data, making the training of a network more difficult [1, 7]. Some authors forgo complexvalued activation functions entirely, choosing instead to scale the complex inputs using bounded real-valued functions which are differentiable with respect to the real and imaginary parts [9, 10, 11]. While this approach allows for more natural grouping of data for classification problems, it requires a modified backpropagation algorithm to train the network, and again the networks are not fully complex-valued. Other authors choose differentiability over boundedness and use elementary transcendental functions [12, 7, 13. Such functions have been used in complex-valued multilayer perceptrons trained using the traditional gradient descent backpropagation algorithm and in other applications [14, 15, 16]. However, the problem of the existence of poles in a bounded region near 0 presents again. Though one can try to scale the data to avoid the regions which contain poles [17], this does not solve the problem, since for unknown composite functions, the locations of poles are not known a priori. The exponential function $\exp (z)$ has been proposed as an alternative to the elementary transcendental functions for some complex-valued neural networks, and experimental evidence suggests better performance of the entire exponential function as activation function than those with poles [18]. 
In this paper, we will derive the backpropagation algorithm for fully complex-valued neural networks based on Newton's method. We compare the performances of using the complex-valued sigmoidal activation function and its Taylor polynomial approximations. Our results give strong supporting evidence for the use of holomorphic functions, in particular polynomial functions, as activation functions for complexvalued neural networks. Polynomials have been used in fully complexvalued functional link networks [19, 20], however their use is limited as activation functions for fully complex-valued multilayer perceptrons. Polynomial functions are differentiable on the entire complex plane and are underlying our computations due to Taylor's Theorem, and they are bounded over any bounded region. Moreover, the complex StoneWeierstrass Theorem implies that any continuous complex-valued function on a compact subset of the complex plane can be approximated by a polynomial [21. Due to the nature of the problems associated with the activation functions in complex-valued neural networks, different choices of activation functions can only suit different types of neural networks properly, and one should only expect an approach to be better than the others in certain applications.

We will allow a more general class of complex-valued functions for activation functions, namely the holomorphic functions. There are two important reasons for this. The first one is that holomorphic functions encompass a general class of functions that are commonly used as activation functions. They allow a wide variety of choices both for activation functions and training methods. The second is that the differentiability of holomorphic functions leads to much simpler formulas in the backpropagation algorithms. For application purpose, we will also consider the backpropagation algorithm using the pseudoNewton's method, since it has computational advantage. Our main results are given by Theorem 4.1, Corollary 5.1, and Theorem 6.1. Theorem 4.1 gives a recursive algorithm to compute the entries of the Hessian matrices in the application of Newton's method to the backpropagation algorithm for complex-valued holomorphic multilayer perceptrons, and Corollary 5.1 gives a recursive algorithm for the application of the pseudo-Newton's method to the backpropagation algorithm based on Theorem 4.1. The recursive algorithms we developed are analogous to the known gradient descent backpropagation algorithm as stated in Section III, hence can be readily implemented in realworld applications. A problem with Newton's method is the choice of steplengths to ensure the algorithm actually converges in applications. Our setting enables us to perform a rigorous analysis for the one-step 
Newton steplength algorithm for the minimization of real-valued complex functions using Newton's method. This is done in Section VI. Our experiments, reported in Section VII, show that the algorithms we developed use significantly fewer iterations to achieve the same results as the gradient descent algorithm. We believe that the Newton's method backpropagation algorithm provides a valuable tool for fast learning for complex-valued neural networks as a practical alternative to the gradient descent methods.

The rest of the paper is as follows. In Section II, we define holomorphic multilayer perceptrons and set up our notation for the network architecture we use throughout the rest of the paper. In Section III, we give a reformulation of the gradient descent backpropagation algorithm based on our setting of holomorphic neural networks. In Section IV, we derive the backpropagation algorithm for holomorphic multilayer perceptrons using Newton's method, and in Section V we restirct the results of Section IV to the pseudo-Newton's method. In Section VI we state the one-step Newton steplength algorithm, and in Section VII we report our experiments. The appendices provide the detailed computations omitted from Section IV and a detailed proof, omitted from Section VI, of the convergence of the one-step Newton steplength algorithm for the minimization of real-valued complex functions.

\section{Holomorphic MLPs: Definition and Network ARCHITECTURE}

A well-used type of artificial neural network is the multilayer perceptron (MLP). An MLP is built of several layers of single neurons hooked together by a network of weight vectors. Usually the activation function is taken to be the same among a single layer of the network; the defining characteristic of the MLP is that in at least one layer, the activation function must be nonlinear. If there is no nonlinear activation function, the network can be collapsed to a two-layer network 22 .

Definition 2.1. A holomorphic MLP is a complex-valued MLP in which the activation function in the layer indexed by $p$ of the network is holomorphic on some domain $\Omega_{p} \subseteq \mathbb{C}$.

Most of the publications on complex-valued neural networks with holomorphic activation functions deal with functions that have poles. We will mainly focus on entire functions for the purpose of applying Newton's method. For these functions, we do not have to worry about the entries of a Hessian matrix hitting the poles. However, we will allow 
some flexibility in our setting and set up our notation for a general $L$ layer holomorphic MLP as follows (see Figure 1).

- The input layer has $m=K_{0}$ input nodes denoted

$$
z_{1}=x_{1}^{(0)}, \ldots, z_{m}=x_{m}^{(0)} .
$$

- There are $L-1$ hidden layers of neurons, and the $p$ th $(1 \leq p \leq$ $L-1)$ hidden layer contains $K_{p}$ nodes. We denote the output of node $j\left(j=1, \ldots, K_{p}\right)$ in the $p$ th layer by $x_{j}^{(p)}$. The inputted weights to the $p$ th layer are denoted $w_{j i}^{(p-1)}\left(j=1, \ldots, K_{p}, i=\right.$ $\left.1, \ldots, K_{p-1}\right)$, where $j$ denotes the target node of the weight in the $p$ th layer and $i$ denotes the source node in the $(p-1)$ th layer. With these conventions we define the weighted net sum and the output of node $j$ of the $p$ th layer by

$$
\left(x_{j}^{(p)}\right)^{\mathrm{net}}=\sum_{i=1}^{K_{p-1}} w_{j i}^{(p-1)} x_{i}^{(p-1)} \text { and } x_{j}^{(p)}=g_{p}\left(\left(x_{j}^{(p)}\right)^{\mathrm{net}}\right),
$$

where $g_{p}$, which is assumed to be holomorphic on some domain $\Omega_{p} \subseteq \mathbb{C}$, is the activation function for all the neurons in the $p$ th layer.
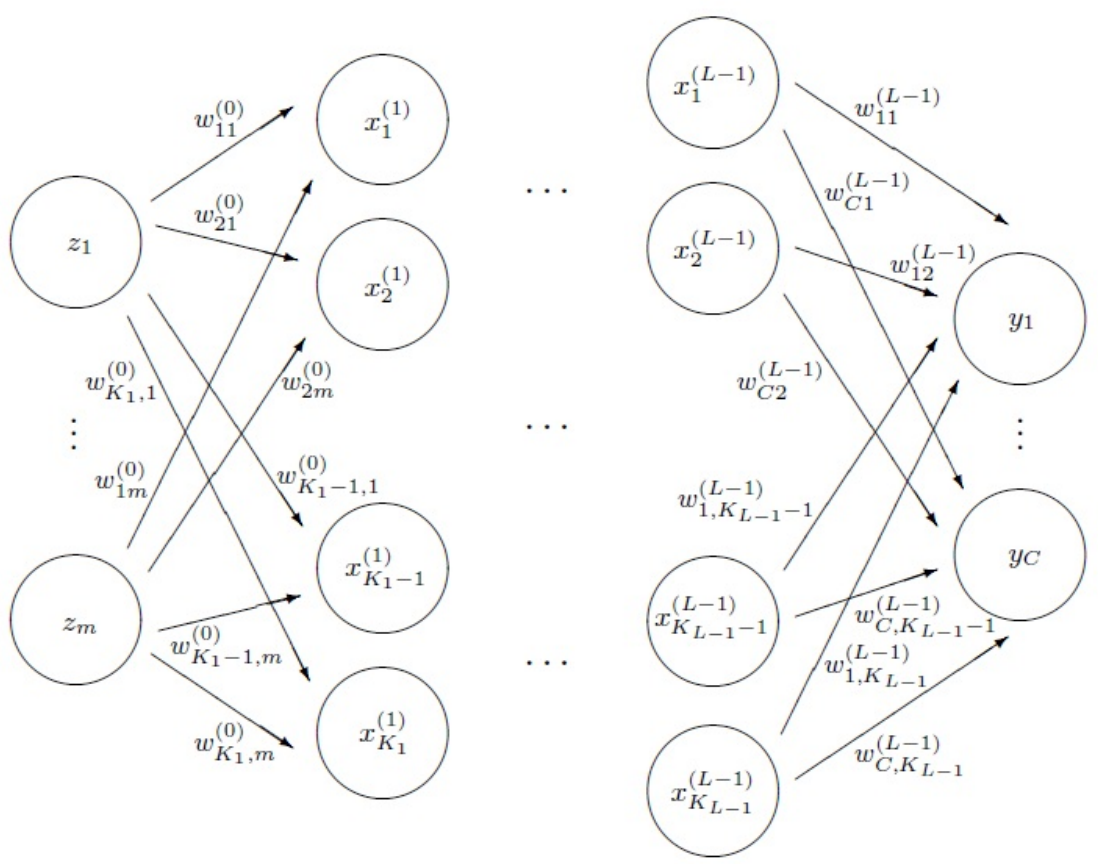

FiguRE 1. Network Architecture 
- The output layer has $C=K_{L}$ output nodes denoted $y_{1}=$ $x_{1}^{(L)}, \ldots, y_{C}=x_{C}^{(L)}$. We define the weighted net sum and the output of node $l(l=1, \ldots, C)$ by

$$
y_{l}^{\text {net }}=\sum_{k=1}^{K_{L-1}} w_{l k}^{(L-1)} x_{k}^{(L-1)} \text { and } y_{l}=g_{L}\left(y_{l}^{\text {net }}\right),
$$

where $g_{L}$, which is assumed to be holomorphic on some domain $\Omega_{L} \subseteq \mathbb{C}$, is the activation function for all the neurons in the output layer.

To train the network, we use a training set with $N$ data points $\left\{\left(z_{t 1}, \ldots, z_{t m}, d_{t 1}, \ldots, d_{t C}\right) \mid t=1, \ldots, N\right\}$, where $\left(z_{t 1}, \ldots, z_{t m}\right)$ is the input vector corresponding to the desired output vector $\left(d_{t 1}, \ldots, d_{t C}\right)$. As the input vector $\left(z_{t 1}, \ldots, z_{t m}\right)$ of the $t$ th training point is propagated throughout the network we update the subscripts of the network calculations with an additional $t$ subscript to signify that those values corre-

spond to the $t$ th training point. For example, $\left(x_{t j}^{(p)}\right)^{\text {net }}, x_{t j}^{(p)}, y_{t l}^{\text {net }}$, and $y_{t l}$. Finally, we train the network by minimizing the standard sum-ofsquares error function

$$
E=\frac{1}{N} \sum_{t=1}^{N} \sum_{l=1}^{C}\left|y_{t l}-d_{t l}\right|^{2}=\frac{1}{N} \sum_{t=1}^{N} \sum_{l=1}^{C}\left(y_{t l}-d_{t l}\right)\left(\overline{y_{t l}}-\overline{d_{t l}}\right) .
$$

\section{The Gradient Descent Backpropagation Algorithm}

Minimization of the error function can be achieved through the use of the backpropagation algorithm. Backpropagation trains the network by updating the output layer weights first in each step (via an update rule from some numerical minimization algorithm), then using the updated output layer weights to update the first hidden layer weights, and so on, "backpropagating" the updates throughout the network until a desired level of accuracy is achieved (usually, this is when the error function drops below a pre-fixed value). In the case of real-valued neural networks, minimization of the error function by Newton's method is generally thought to be too computationally "expensive," and several different methods are commonly used to approximate the Hessian matrices instead of computing them directly: for example the conjugate gradient, truncated Newton, Gauss-Newton and Levenberg-Marquardt algorithms [23, 24, 25, 3, 26]. In contrast, for complex-valued neural networks, gradient descent methods, which are known to give stable (albeit slow) convergence, are commonly used due to their relatively simple formulations, and a number of such minimization algorithms exist [27, 17, 28]. 
We reformulate a backpropagation algorithm using gradient descent according to our setting of the neural networks defined in Section II for two reasons: the algorithm has a much simpler formulation compared with the known ones [22, 17] due to the activation functions being taken to be holomorphic, and we will use it for comparison purpose. A similar formulation of the backpropagation algorithm to ours is presented in [29]. The formulas of gradient descent for complex functions can be found in [30]. We use the following vector notation. For $1 \leq p \leq L$, we denote the weights that input into the $p$ th layer of the network using a vector whose components correspond to the target nodes:

$$
\mathbf{w}^{(p-1)}:=\left(w_{11}^{(p-1)}, \ldots, w_{1 K_{p-1}}^{(p-1)}, \ldots, w_{K_{p} 1}^{(p-1)}, \ldots, w_{K_{p} K_{p-1}}^{(p-1)}\right)^{T}
$$

that is, the components of $\mathbf{w}^{(p-1)}$ are

$$
\mathbf{w}^{(p-1)}\left[(j-1) \cdot K_{p-1}+i\right]=w_{j i}^{(p-1)},
$$

where $j=1, \ldots, K_{p}, i=1, \ldots, K_{p-1}$. Using this notation the update steps for backpropagation look like

$$
\mathbf{w}^{(p-1)}(n+1)=\mathbf{w}^{(p-1)}(n)+\mu(n) \Delta \mathbf{w}^{(p-1)},
$$

where $\mathbf{w}^{(p-1)}(n)$ denotes the weight value after the $n$th iteration of the training algorithm, and $\mu(n)$ denotes the learning rate or steplength which is allowed to vary with each iteration.

Using the gradient descent method, the update for the $(p-1)$ th layer of a holomorphic complex-valued neural network is ([30], p. 60)

$$
\Delta \mathbf{w}^{(p-1)}=-\left(\frac{\partial E}{\partial \mathbf{w}^{(p-1)}}\right)^{*}
$$

Suppose the activation function for the $p$ th layer of the network, $p=$ $1, \ldots, L$, satisfies

$$
\overline{g(z)}=g(\bar{z})
$$

Coordinate-wise the partial derivatives $\frac{\partial E}{\partial w_{l k}^{(L-1)}}$, taken with respect to the output layer weights $w_{l k}^{(L-1)}, l=1, \ldots, C, k=1, \ldots, K_{L-1}$, are given 
by

$$
\begin{aligned}
\frac{\partial E}{\partial w_{l k}^{(L-1)}} & =\frac{\partial}{\partial w_{l k}^{(L-1)}}\left[\frac{1}{N} \sum_{t=1}^{N} \sum_{h=1}^{C}\left(y_{t h}-d_{t h}\right)\left(\overline{y_{t h}}-\overline{y_{t h}}\right)\right] \\
& =\frac{1}{N} \sum_{t=1}^{N}\left[\frac{\partial y_{t l}}{\partial w_{l k}^{(L-1)}}\left(\overline{y_{t l}}-\overline{d_{t l}}\right)+\left(y_{t l}-d_{t l}\right) \frac{\partial \overline{y_{t l}}}{\partial w_{l k}^{(L-1)}}\right] \\
& =\frac{1}{N} \sum_{t=1}^{N}\left(\frac{\partial y_{t l}}{\partial y_{t l}^{\text {net }}} \frac{\partial y_{t l}^{\text {net }}}{\partial w_{l k}^{(L-1)}}+\frac{\partial y_{t l}}{\partial \overline{y_{t l}^{\text {net }}}} \frac{\partial \overline{y_{t l}^{\text {net }}}}{\partial w_{l k}^{(L-1)}}\right)\left(\overline{y_{t l}}-\overline{d_{t l}}\right) \\
& =\frac{1}{N} \sum_{t=1}^{N}\left(\overline{y_{t l}}-\overline{d_{t l}}\right) g_{L}^{\prime}\left(y_{t l}^{\text {net }}\right) x_{t k}^{(L-1)},
\end{aligned}
$$

so that

$$
\left(\frac{\partial E}{\partial w_{l k}^{(L-1)}}\right)^{*}=\frac{1}{N} \sum_{t=1}^{N}\left(y_{t l}-d_{t l}\right) g_{L}^{\prime}\left(\overline{y_{t l}^{\mathrm{net}}}\right) \overline{x_{t k}^{(L-1)}}
$$

The partial derivatives $\left(\frac{\partial E}{\partial w_{j i}^{(p-1)}}\right)^{*}$, taken with respect to the hidden layer weights $w_{j i}^{(p-1)}, 1 \leq p \leq L-1, j=1, \ldots, K_{p}, i=1, \ldots, K_{p-1}$, are computed recursively. The partial derivatives $\frac{\partial E}{\partial w_{j i}^{(L-2)}}$, taken with respect to the $(L-2)$ th hidden layer weights, are computed using the updated $(L-1)$ th output layer weights:

$$
\begin{aligned}
\frac{\partial E}{\partial w_{j i}^{(L-2)}} & =\frac{\partial}{\partial w_{j i}^{(L-2)}}\left[\frac{1}{N} \sum_{t=1}^{N} \sum_{l=1}^{C}\left(y_{t l}-d_{t l}\right)\left(\overline{y_{t l}}-\overline{y_{t l}}\right)\right] \\
& =\frac{1}{N} \sum_{t=1}^{N} \sum_{l=1}^{C}\left[\frac{\partial y_{t l}}{\partial w_{j i}^{(L-2)}}\left(\overline{y_{t l}}-\overline{d_{t l}}\right)+\left(y_{t l}-d_{t l}\right) \frac{\partial \overline{y_{t l}}}{\partial w_{j i}^{(L-2)}}\right]
\end{aligned}
$$


where

$$
\begin{aligned}
\frac{\partial y_{t l}}{\partial w_{j i}^{(L-2)}} & =\frac{\partial y_{t l}}{\partial y_{t l}^{\mathrm{net}}} \frac{\partial y_{t l}^{\mathrm{net}}}{\partial w_{j i}^{(L-2)}}+\frac{\partial y_{t l}}{\partial \overline{y_{t l}^{\mathrm{net}}}} \frac{\overline{\partial y_{t l}^{\mathrm{net}}}}{\partial w_{j i}^{(L-2)}} \\
& =g_{L}^{\prime}\left(y_{t l}^{\mathrm{net}}\right)\left(\frac{\partial y_{t l}^{\mathrm{net}}}{\partial x_{t j}^{(L-1)}} \frac{\partial x_{t j}^{(L-1)}}{\partial w_{j i}^{(L-2)}}+\frac{\partial y_{t l}^{\mathrm{net}}}{\partial \overline{x_{t j}^{(L-1)}}} \frac{\partial \overline{x_{t j}^{(L-1)}}}{\partial w_{j i}^{(L-2)}}\right) \\
& =g_{L}^{\prime}\left(y_{t l}^{\mathrm{net}}\right) w_{l j}^{(L-1)}\left(\frac{\partial x_{t j}^{(L-1)}}{\partial\left(x_{t j}^{(L-1)}\right)^{\mathrm{net}}} \frac{\partial\left(x_{t j}^{(L-1)}\right)^{\mathrm{net}}}{\partial w_{j i}^{(L-2)}}\right. \\
& =g_{L}^{\prime}\left(y_{t l}^{\mathrm{net}}\right) w_{l j}^{(L-1)} g_{L-1}^{\prime}\left(\left(x_{t j}^{(L-1)}\right)^{\mathrm{net}}\right)^{(L-2)} x_{t i}^{(L-1)}
\end{aligned}
$$

and

$$
\begin{aligned}
\frac{\partial \overline{y_{t l}}}{\partial w_{j i}^{(L-2)}} & =\frac{\partial \overline{y_{t l}}}{\partial \overline{y_{t l}^{\text {net }}}} \frac{\overline{\partial y_{t l}^{\text {net }}}}{\partial w_{j i}^{(L-2)}}+\frac{\partial \overline{y_{t l}}}{\partial y_{t l}^{\text {net }}} \frac{\partial y_{t l}^{\text {net }}}{\partial w_{j i}^{(L-2)}} \\
& =g_{L}^{\prime}\left(\overline{y_{t l}^{\text {net }}}\right)\left(\frac{\partial \overline{y_{t l}^{\text {net }}}}{\partial \overline{x_{t j}^{(L-1)}}} \frac{\partial \overline{x_{t j}^{(L-1)}}}{\partial w_{j i}^{(L-2)}}+\frac{\partial \overline{y_{t l}^{\text {net }}}}{\partial x_{t j}^{(L-1)}} \frac{\partial x_{t j}^{(L-1)}}{\partial w_{j i}^{(L-2)}}\right) \\
& =g_{L}^{\prime}\left(\overline{y_{t l}^{\text {net }}}\right) \overline{w_{l j}^{(L-1)}}\left(\frac{\partial \overline{x_{t j}^{(L-1)}}}{\partial \overline{\left(x_{t j}^{(L-1)}\right)^{\text {net }}}} \frac{\partial\left(x_{t j}^{(L-1)}\right)^{\text {net }}}{\partial w_{j i}^{(L-2)}}\right. \\
+\frac{\partial\left(x_{t j}^{(L-1)}\right)^{\mathrm{net}}}{\partial x_{t j}^{(L-1)}} & \partial\left(x_{t j}^{(L-1)}\right)^{\text {net }} \\
& =0
\end{aligned}
$$

so that

$$
\begin{array}{r}
\left(\frac{\partial E}{\partial w_{j i}^{(L-2)}}\right)^{*}=\frac{1}{N} \sum_{t=1}^{N}\left(\sum_{l=1}^{C}\left(y_{t l}-d_{t l}\right) g_{L}^{\prime}\left(\overline{y_{t l}^{\mathrm{net}}}\right) \overline{w_{l j}^{(L-1)}}\right) \\
\cdot g_{L-1}^{\prime}\left(\overline{\left.\left(x_{t j}^{(L-1)}\right)^{\mathrm{net}}\right) \overline{x_{t i}^{(L-2)}}}\right.
\end{array}
$$


and so on. We summarize the partial derivatives by

$$
\left(\frac{\partial E}{\partial w_{j i}^{(p-1)}}\right)^{*}=\frac{1}{N} \sum_{t=1}^{N} E_{t j}^{(p)} \overline{x_{t i}^{(p-1)}}
$$

$1 \leq p \leq L$, where $j=1, \ldots, K_{p}, i=1, \ldots, K_{p-1}$, and the $E_{t j}^{(p)}$ are given recursively by

$$
E_{t l}^{(L)}=\left(y_{t l}-d_{t l}\right) g_{L}^{\prime}\left(\overline{y_{t l}^{\mathrm{net}}}\right)
$$

where $l=1, \ldots, C, t=1, \ldots, N$; and for $1 \leq p \leq L-1$,

$$
E_{t j}^{(p)}=\left[\sum_{\alpha=1}^{K_{p+1}} E_{t \alpha}^{(p+1)} \overline{w_{\alpha j}^{(p)}}\right] g_{p}^{\prime}\left(\overline{\left(x_{t j}^{(p)}\right)^{\mathrm{net}}}\right),
$$

where $j=1, \ldots, K_{p}, t=1, \ldots, N$. The gradient descent method is well known to be rather slow in the convergence of the error function. We next derive formulas for the backpropagation algorithm using Newton's method (compare with [22, 17]).

\section{Backpropagation Using NeWton's Method}

The weight updates for Newton's method with complex functions are given by formula (111) of [30] (we omit the superscripts, which index the layers, to simplify our writing):

$$
\Delta \mathbf{w}=\left(\mathcal{H}_{\mathbf{w w}}-\mathcal{H}_{\overline{\mathbf{w} w}} \mathcal{H}_{\overline{\mathbf{w} w}}^{-1} \mathcal{H}_{\mathbf{w} \overline{\mathbf{w}}}\right)^{-1}\left[\mathcal{H}_{\overline{\mathbf{w}} \mathbf{w}} \mathcal{H}_{\overline{\mathbf{w} w}}^{-1}\left(\frac{\partial E}{\partial \overline{\mathbf{w}}}\right)^{*}-\left(\frac{\partial E}{\partial \mathbf{w}}\right)^{*}\right] .
$$

To apply the Newton algorithm we need to compute the Hessian matrices (again omitting the superscripts)

$$
\mathcal{H}_{\mathbf{w w}}=\frac{\partial}{\partial \mathbf{w}}\left(\frac{\partial E}{\partial \mathbf{w}}\right)^{*} \text { and } \mathcal{H}_{\overline{\mathbf{w}} \mathbf{w}}=\frac{\partial}{\partial \overline{\mathbf{w}}}\left(\frac{\partial E}{\partial \mathbf{w}}\right)^{*},
$$

where the entries of $\left(\frac{\partial E}{\partial \mathbf{w}}\right)^{*}$ are given by 3.3 . Note that although 4.1 asks for the four Hessian matrices $\mathcal{H}_{\mathbf{w w}}, \mathcal{H}_{\overline{\mathbf{w}} \mathbf{w}}, \mathcal{H}_{\mathbf{w} \overline{\mathbf{w}}}$, and $\mathcal{H}_{\overline{\mathrm{ww}}}$, we have $\mathcal{H}_{\mathrm{w} \overline{\mathbf{w}}}=\overline{\mathcal{H}_{\overline{\mathrm{w}} \mathbf{w}}}$ and $\mathcal{H}_{\overline{\mathrm{ww}}}=\overline{\mathcal{H}}_{\mathrm{ww}}$. Thus we only need to compute two of them.

We consider the entries of the Hessian matrices $\mathcal{H}_{\mathbf{w w}}$ and $\mathcal{H}_{\overline{\mathbf{w}} \mathbf{w}}$. For the $(p-1)$ th layer, the entries of $\mathcal{H}_{\mathbf{w w}}$ are given by (see $\left.(3.1)\right)$

$$
\mathcal{H}_{\mathbf{w w}}\left[(j-1) \cdot K_{p-1}+i,(b-1) \cdot K_{p-1}+a\right]=\frac{\partial}{\partial w_{b a}^{(p-1)}}\left(\frac{\partial E}{\partial w_{j i}^{(p-1)}}\right)^{*}
$$


where $j, b=1, \ldots, K_{p}$ and $i, a=1, \ldots, K_{p-1}$, and the entries of $\mathcal{H}_{\overline{\overline{\mathbf{w}}} \mathbf{w}}$ are given by

$$
\mathcal{H}_{\overline{\mathbf{w}} \mathbf{w}}\left[(j-1) \cdot K_{p-1}+i,(b-1) \cdot K_{p-1}+a\right]=\frac{\partial}{\partial \overline{w_{b a}^{(p-1)}}}\left(\frac{\partial E}{\partial w_{j i}^{(p-1)}}\right)^{*}
$$

where $j, b=1, \ldots, K_{p}$ and $i, a=1, \ldots, K_{p-1}$.

First we derive an explicit formula for the entries of the Hessians $\mathcal{H}_{\mathbf{w w}}$. We start with the output layer and compute $\frac{\partial}{\partial w_{k q}^{(L-1)}}\left(\frac{\partial E}{\partial w_{l p}^{(L-1)}}\right)^{*}$, where $k, l=1, \ldots, C$ and $q, p=1, \ldots, K_{L-1}$. Observe that if $k \neq l$, then each term $\left(y_{t l}-d_{t l}\right) g_{L}^{\prime}\left(\overline{y_{t l}^{\text {net }}}\right) \overline{x_{t p}^{(L-1)}}$ in the cogradient given by 3.3 and 3.4 does not depend on the weights $w_{k q}^{(L-1)}$, hence this entry of the Hessian will be 0. So the Hessian matrix for the output layer has a block diagonal form:

$\mathcal{H}_{\mathbf{w}^{(L-1)} \mathbf{w}^{(L-1)}}=\operatorname{diag}\left\{\left[\frac{\partial}{\partial w_{l q}^{(L-1)}}\left(\frac{\partial E}{\partial w_{l p}^{(L-1)}}\right)^{*}\right]_{\substack{1 \leq p \leq K_{L-1} \\ 1 \leq q \leq K_{L-1}}}: l=1, \ldots, C\right\}$.

Now:

$$
\begin{array}{r}
\frac{\partial}{\partial w_{l q}^{(L-1)}}\left(\frac{\partial E}{\partial w_{l p}^{(L-1)}}\right)^{*}=\frac{\partial}{\partial w_{l q}^{(L-1)}}\left[\frac{1}{N} \sum_{t=1}^{N}\left(y_{t l}-d_{t l}\right) g_{L}^{\prime}\left(\overline{y_{t l}^{\mathrm{net}}}\right) \overline{x_{t p}^{(L-1)}}\right] \\
=\frac{1}{N} \sum_{t=1}^{N}\left[\left(y_{t l}-d_{t l}\right) \frac{\partial g_{L}^{\prime}\left(\overline{y_{t l}^{\mathrm{net}}}\right)}{\partial w_{l q}^{(L-1)}}+g_{L}^{\prime}\left(\overline{y_{t l}^{\mathrm{net}}}\right) \frac{\partial y_{t l}}{\partial w_{l q}^{(L-1)}}\right] \overline{x_{t p}^{(L-1)}}
\end{array}
$$

where

$$
\frac{\partial y_{t l}}{\partial w_{l q}^{(L-1)}}=\frac{\partial y_{t l}}{\partial y_{t l}^{\mathrm{net}}} \frac{\partial y_{t l}^{\mathrm{net}}}{\partial w_{l q}^{(L-1)}}+\frac{\partial y_{t l}}{\partial \overline{y_{t l}^{\mathrm{net}}}} \frac{\partial \overline{y_{t l}^{\mathrm{net}}}}{\partial w_{l q}^{(L-1)}}=g_{L}^{\prime}\left(y_{t l}^{\mathrm{net}}\right) x_{t q}^{(L-1)}
$$

since $g_{L}$ is holomorphic and therefore $\frac{\partial y_{t l}}{\partial y_{t l}^{\text {net }}}=0$ (Cauchy-Riemann condition), and similarly

$$
\frac{\partial g_{L}^{\prime}\left(\overline{y_{t l}^{\mathrm{net}}}\right)}{\partial w_{l q}^{(L-1)}}=\frac{\partial g_{L}^{\prime}\left(\overline{y_{t l}^{\mathrm{net}}}\right)}{\partial \overline{y_{t l}^{\mathrm{net}}}} \frac{\overline{\partial y_{t l}^{\mathrm{net}}}}{\partial w_{l q}^{(L-1)}}+\frac{\partial g_{L}^{\prime}\left(\overline{y_{t l}^{\mathrm{net}}}\right)}{\partial y_{t l}^{\mathrm{net}}} \frac{\partial y_{t l}^{\mathrm{net}}}{\partial w_{l q}^{(L-1)}}=0 .
$$


Combining these two partial derivatives with (4.3) gives the following formula for the entries of the output layer Hessian matrix:

$$
\begin{aligned}
& \frac{\partial}{\partial w_{k q}^{(L-1)}}\left(\frac{\partial E}{\partial w_{l p}^{(L-1)}}\right)^{*} \\
& \quad= \begin{cases}\frac{1}{N} \sum_{t=1}^{N} g_{L}^{\prime}\left(\overline{y_{t l}^{\text {net }}}\right) g_{L}^{\prime}\left(y_{t l}^{\text {net }}\right) \overline{x_{t p}^{(L-1)}} x_{t q}^{(L-1)} & \text { if } k=l, \\
0 & \text { if } k \neq l\end{cases}
\end{aligned}
$$

After updating the output layer weights, the backpropagation algorithm updates the hidden layer weights recursively. We compute the entries of the Hessian $\mathcal{H}_{\mathbf{w}^{(p-1)} \mathbf{w}^{(p-1)}}$ for the $(p-1)$ th layer using $(3.3)$ :

$$
\begin{aligned}
\frac{\partial}{\partial w_{b a}^{(p-1)}}\left(\frac{\partial E}{\partial w_{j i}^{(p-1)}}\right)^{*} & =\frac{\partial}{\partial w_{b a}^{(p-1)}}\left[\frac{1}{N} \sum_{t=1}^{N} E_{t j}^{(p)} \overline{x_{t i}^{(p-1)}}\right] \\
& =\frac{1}{N} \sum_{t=1}^{N} \frac{\partial E_{t j}^{(p)}}{\partial w_{b a}^{(p-1)}} \overline{x_{t i}^{(p-1)}}
\end{aligned}
$$

Applying the chain rule to (3.5), we have

$$
\begin{aligned}
& \frac{\partial E_{t j}^{(p)}}{\partial w_{b a}^{(p-1)}}=\frac{\partial}{\partial w_{b a}^{(p-1)}}\left[\left(\sum_{\eta=1}^{K_{p+1}} E_{t \eta}^{(p+1)} \overline{w_{\eta j}^{(p)}}\right) g_{p}^{\prime}\left(\overline{\left(x_{t j}^{(p)}\right)^{\mathrm{net}}}\right)\right] \\
& =g_{p}^{\prime}\left(\overline{\left(x_{t j}^{(p)}\right)^{\mathrm{net}}}\right) \sum_{\eta=1}^{K_{p+1}} \frac{\partial E_{t \eta}^{(p+1)}}{\partial w_{b a}^{(p-1)}} \overline{w_{\eta j}^{(p)}} \\
& =g_{p}^{\prime}\left(\overline{\left(x_{t j}^{(p)}\right)^{\mathrm{net}}}\right) \sum_{\eta=1}^{K_{p+1}}\left[\frac{\partial E_{t \eta}^{(p+1)}}{\partial x_{t b}^{(p)}} \frac{\partial x_{t b}^{(p)}}{\partial w_{b a}^{(p-1)}}+\frac{\partial E_{t \eta}^{(p+1)}}{\partial \overline{x_{t b}^{(p)}}} \frac{\partial \overline{x_{t b}^{(p)}}}{\partial w_{b a}^{(p-1)}}\right] \overline{w_{\eta j}^{(p)}} \\
& =g_{p}^{\prime}\left(\overline{\left(x_{t j}^{(p)}\right)^{\text {net }}}\right) \sum_{\eta=1}^{K_{p+1}} \frac{\partial E_{t \eta}^{(p+1)}}{\partial x_{t b}^{(p)}}\left[\frac{\partial x_{t b}^{(p)}}{\partial\left(x_{t b}^{(p)}\right)^{\text {net }}} \frac{\partial\left(x_{t b}^{(p)}\right)^{\text {net }}}{\partial w_{b a}^{(p-1)}}\right. \\
& \left.+\frac{\partial x_{t b}^{(p)}}{\partial \overline{\left(x_{t b}^{(p)}\right)^{\text {net }}}} \frac{\partial \overline{\left(x_{t b}^{(p)}\right)^{\text {net }}}}{\partial w_{b a}^{(p-1)}}\right] \overline{w_{\eta j}^{(p)}} \\
& =g_{p}^{\prime}\left(\overline{\left(x_{t j}^{(p)}\right)^{\mathrm{net}}}\right) \sum_{\eta=1}^{K_{p+1}} \frac{\partial E_{t \eta}^{(p+1)}}{\partial x_{t b}^{(p)}} g_{p}^{\prime}\left(\left(x_{t b}^{(p)}\right)^{\mathrm{net}}\right) x_{t a}^{(p-1)} \overline{w_{\eta j}^{(p)}} .
\end{aligned}
$$


In the above computation, we have used the fact that $g_{p}$ is holomorphic and hence $\frac{\partial g_{p}^{\prime}\left(\overline{\left(x_{t j}^{(p)}\right)^{\mathrm{net}}}\right)}{\partial w_{b a}^{(p-1)}}=0$ and $\frac{\partial \overline{x_{t b}^{(p)}}}{\partial w_{b a}^{(p-1)}}=0, \frac{\partial x_{t b}^{(p)}}{\partial\left(x_{t b}^{(p)}\right)^{\mathrm{net}}}=0$, and $\frac{\partial \overline{\left(x_{t b}^{(p)}\right)^{\mathrm{net}}}}{\partial w_{b a}^{(p-1)}}=0$. Combining 4.5 and 4.6 , we have:

$$
\begin{aligned}
& \frac{\partial}{\partial w_{b a}^{(p-1)}}\left(\frac{\partial E}{\partial w_{j i}^{(p-1)}}\right)^{*}=\frac{1}{N} \sum_{t=1}^{N} {\left[\sum_{\eta=1}^{K_{p+1}} \frac{\partial E_{t \eta}^{(p+1)}}{\partial x_{t b}^{(p)}} \overline{w_{\eta j}^{(p)}}\right] } \\
& \cdot g_{p}^{\prime}\left(\overline{\left.\left(x_{t j}^{(p)}\right)^{\mathrm{net}}\right)} g_{p}^{\prime}\left(\left(x_{t b}^{(p)}\right)^{\mathrm{net}}\right) \overline{x_{t i}^{(p-1)}} x_{t a}^{(p-1)} .\right.
\end{aligned}
$$

Next, we derive a recursive rule for finding the partial derivatives $\frac{\partial E_{t \eta}^{(p+1)}}{\partial x_{t b}^{(p)}}$. For computational purposes, an explicit formula for $\frac{\partial E_{t \eta}^{(L)}}{\partial x_{t b}^{(L-1)}}$ is not necessary. What we need is a recursive formula for these partial derivatives as will be apparent shortly. Using (3.5) we have the following:

$$
\begin{aligned}
& \frac{\partial E_{t \eta}^{(p+1)}}{\partial x_{t b}^{(p)}}=\frac{\partial}{\partial x_{t b}^{(p)}}\left[\left(\sum_{\alpha=1}^{K_{p+2}} E_{t \alpha}^{(p+2)} \overline{w_{\alpha \eta}^{(p+1)}}\right) g_{p+1}^{\prime}\left(\overline{\left(x_{t \eta}^{(p+1)}\right)^{\text {net }}}\right)\right] \\
& =g_{p+1}^{\prime}\left(\overline{\left(x_{t \eta}^{(p+1)}\right)^{\mathrm{net}}}\right) \sum_{\alpha=1}^{K_{p+2}} \frac{\partial E_{t \alpha}^{(p+2)}}{\partial x_{t b}^{(p)}} \overline{w_{\alpha \eta}^{(p+1)}} \\
& =g_{p+1}^{\prime}\left(\overline{\left(x_{t \eta}^{(p+1)}\right)^{\mathrm{net}}}\right) \sum_{\alpha=1}^{K_{p+2}} \sum_{\beta=1}^{K_{p+1}}\left[\frac{\partial E_{t \alpha}^{(p+2)}}{\partial x_{t \beta}^{(p+1)}} \frac{\partial x_{t \beta}^{(p+1)}}{\partial x_{t b}^{(p)}}\right. \\
& \left.+\frac{\partial E_{t \alpha}^{(p+2)}}{\partial \overline{x_{t \beta}^{(p+1)}}} \frac{\partial \overline{x_{t \beta}^{(p+1)}}}{\partial x_{t b}^{(p)}}\right] \overline{w_{\alpha \eta}^{(p+1)}}
\end{aligned}
$$




$$
\begin{aligned}
& =g_{p+1}^{\prime}\left(\overline{\left(x_{t \eta}^{(p+1)}\right)^{\mathrm{net}}}\right) \sum_{\alpha=1}^{K_{p+2}} \sum_{\beta=1}^{K_{p+1}} \frac{\partial E_{t \alpha}^{(p+2)}}{\partial x_{t \beta}^{(p+1)}} \\
& \cdot\left[\frac{\partial x_{t \beta}^{(p+1)}}{\partial\left(x_{t \beta}^{(p+1)}\right)^{\mathrm{net}}} \frac{\partial\left(x_{t \beta}^{(p+1)}\right)^{\mathrm{net}}}{\partial x_{t b}^{(p)}}+\frac{\partial x_{t \beta}^{(p+1)}}{\partial \overline{\left(x_{t \beta}^{(p+1)}\right)^{\mathrm{net}}}} \frac{\partial \overline{\left(x_{t \beta}^{(p+1)}\right)^{\mathrm{net}}}}{\partial x_{t b}^{(p)}}\right] \overline{w_{\alpha \eta}^{(p+1)}} \\
& =\sum_{\beta=1}^{K_{p+1}}\left[\sum_{\alpha=1}^{K_{p+2}} \frac{\partial E_{t \alpha}^{(p+2)}}{\partial x_{t \beta}^{(p+1)}} \overline{w_{\alpha \eta}^{(p+1)}}\right] \\
& \cdot g_{p+1}^{\prime}\left(\overline{\left(x_{t \eta}^{(p+1)}\right)^{\mathrm{net}}}\right) g_{p+1}^{\prime}\left(\left(x_{t \beta}^{(p+1)}\right)^{\mathrm{net}}\right) w_{\beta b}^{(p)} \text {. }
\end{aligned}
$$

This gives a recursive formula for computing the partial derivatives $\frac{\partial E_{t \eta}^{(p+1)}}{\partial x_{t b}^{(p)}}$. We will combine the above calculations to give a more concise recursive algorithm for computing the entries of the matrices $\mathcal{H}_{\mathbf{w w}}$ in Theorem 4.1, below.

Next we consider the Hessians $\mathcal{H}_{\overline{\mathbf{w}} \mathbf{w}}$. Again we start with the output layer and compute $\frac{\partial}{\partial \overline{w_{k q}^{(L-1)}}}\left(\frac{\partial E}{\partial w_{l p}^{(L-1)}}\right)^{*}$. Using the fact that $\frac{\partial E}{\partial w_{l p}^{(L-1)}}$ does not depend on $\overline{w_{k q}^{(L-1)}}$ if $k \neq l$, we see that the output layer Hessian $\mathcal{H}{\overline{\mathbf{w}^{(L-1)}}}_{\mathbf{w}^{(L-1)}}$ is also block diagonal with blocks

$$
\left[\frac{\partial}{\partial \overline{w_{l q}^{(L-1)}}}\left(\frac{\partial E}{\partial w_{l p}^{(L-1)}}\right)^{*}\right]_{\substack{1 \leq p \leq K_{L-1} \\ 1 \leq q \leq K_{L-1}}}
$$

for $l=1, \ldots, C$. Computing the entries in these blocks,

$$
\begin{array}{r}
\frac{\partial}{\partial \overline{w_{l q}^{(L-1)}}}\left(\frac{\partial E}{\partial w_{l p}^{(L-1)}}\right)^{*}=\frac{\partial}{\partial \overline{w_{l q}^{(L-1)}}}\left[\frac{1}{N} \sum_{t=1}^{N}\left(y_{t l}-d_{t l}\right) g_{L}^{\prime}\left(\overline{y_{t l}^{\mathrm{net}}}\right) \overline{x_{t p}^{(L-1)}}\right] \\
\quad=\frac{1}{N} \sum_{t=1}^{N}\left[\left(y_{t l}-d_{t l}\right) \frac{\partial g_{L}^{\prime}\left(\overline{y_{t l}^{\mathrm{net}}}\right)}{\partial \overline{w_{l q}^{(L-1)}}}+g_{L}^{\prime}\left(\overline{y_{t l}^{\mathrm{net}}}\right) \frac{\partial y_{t l}}{\partial \overline{w_{l q}^{(L-1)}}}\right] \overline{x_{t p}^{(L-1)}}
\end{array}
$$

where $\frac{\partial y_{t l}}{\partial w_{l q}^{(L-1)}}=0$, and

$$
\begin{aligned}
\frac{\partial g_{L}^{\prime}\left(\overline{y_{t l}^{\text {net }}}\right)}{\partial \overline{w_{l q}^{(L-1)}}} & =\frac{\partial g_{L}^{\prime}\left(\overline{y_{t l}^{\text {net }}}\right)}{\partial \overline{y_{t l}^{\text {net }}}} \frac{\partial \overline{y_{t l}^{\text {net }}}}{\partial \overline{w_{l q}^{(L-1)}}}+\frac{\partial g_{L}^{\prime}\left(\overline{y_{t l}^{\text {net }}}\right)}{\partial y_{t l}^{\text {net }}} \frac{\partial y_{t l}^{\text {net }}}{\partial \overline{w_{l q}^{(L-1)}}} \\
& =g_{L}^{\prime \prime}\left(\overline{y_{t l}^{\text {net }}}\right) \overline{x_{t q}^{(L-1)}} .
\end{aligned}
$$


Thus:

$$
\begin{aligned}
\frac{\partial}{\partial \overline{w_{k q}^{(L-1)}}} & \left(\frac{\partial E}{\partial w_{l p}^{(L-1)}}\right)^{*} \\
& = \begin{cases}\frac{1}{N} \sum_{t=1}^{N}\left(y_{t l}-d_{t l}\right) g_{L}^{\prime \prime}\left(\overline{y_{t l}^{\text {net }}}\right) \overline{x_{t q}^{(L-1)} x_{t p}^{(L-1)}} & \text { if } k=l, \\
0 & \text { if } k \neq l .\end{cases}
\end{aligned}
$$

The entries of the Hessian $\mathcal{H}_{\mathbf{w}^{(p-1)} \mathbf{w}^{(p-1)}}$ for the $(p-1)$ th layer can be computed similarly. We record the formula here and provide the detailed computations in Appendix A. We have:

$$
\begin{aligned}
& \frac{\partial}{\partial \overline{w_{b a}^{(p-1)}}}\left(\frac{\partial E}{\partial w_{j i}^{(p-1)}}\right)^{*}
\end{aligned}
$$

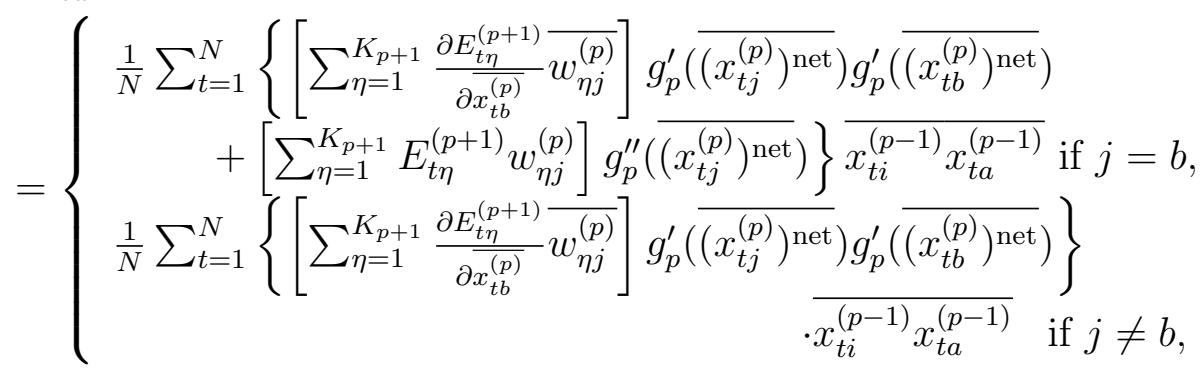

where $j, b=1, \ldots, K_{p}$ and $i, a=1, \ldots, K_{p+1}$, and the partial derivatives $\frac{\partial E_{t \eta}^{(p+1)}}{\partial \overline{x_{t b}^{(p)}}}$ are given recursively by

$$
\begin{aligned}
& \frac{\partial E_{t \eta}^{(p+1)}}{\partial \overline{x_{t b}^{(p)}}}=\sum_{\beta=1}^{K_{p+1}} {\left[\sum_{\alpha=1}^{K_{p+2}} \frac{\partial E_{t \alpha}^{(p+2)}}{\partial \overline{x_{t \beta}^{(p+1)}}} \overline{w_{\alpha \eta}^{(p+1)}}\right] } \\
& \cdot g_{p+1}^{\prime}\left(\overline{\left(x_{t \eta}^{(p+1)}\right)^{\text {net }}}\right) g_{p+1}^{\prime}\left(\overline{\left.\left(x_{t \beta}^{(p+1)}\right)^{\text {net }}\right) \overline{w_{\beta b}^{(p)}}}\right. \\
&+\left[\sum_{\alpha=1}^{K_{p+2}} E_{t \alpha}^{(p+2)} \overline{w_{\alpha \eta}^{(p+1)}}\right] g_{p+1}^{\prime \prime}\left(\overline{\left(x_{t \eta}^{(p+1)}\right)^{\text {net }}} \overline{w_{\eta b}^{(p)}} .\right.
\end{aligned}
$$

We now summarize the formulas we have derived in the following theorem.

Theorem 4.1 (Newton Backpropagation Algorithm for Holomorphic Neural Networks). The weight updates for the holomorphic MLPs with 
activation functions satisfying

$$
\overline{g(z)}=g(\bar{z})
$$

$p=1, \ldots, L$, using the backpropagation algorithm with Newton's method are given by

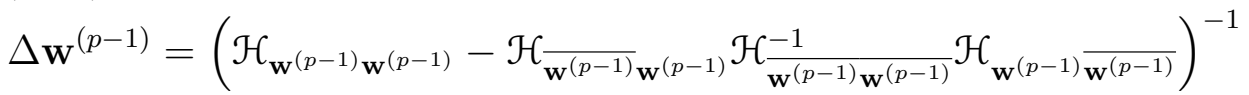

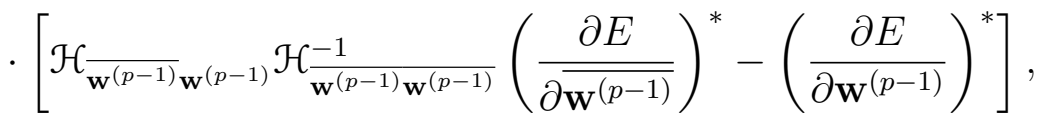

where:

(1) the entries of the Hessian matrices $\mathcal{H}_{\mathbf{w}^{(p-1)} \mathbf{w}^{(p-1)}}$ for $p=1, \ldots, L$ are given by

$$
\frac{\partial}{\partial w_{b a}^{(p-1)}}\left(\frac{\partial E}{\partial w_{j i}^{(p-1)}}\right)^{*}=\frac{1}{N} \sum_{t=1}^{N} \gamma_{t j b}^{(p)} \overline{x_{t i}^{(p-1)}} x_{t a}^{(p-1)}
$$

for $j, b=1, \ldots, K_{p}$ and $i, a=1, \ldots, K_{p-1}$, where the $\gamma_{t j b}^{(p)}$ are defined for $t=1, \ldots, N$ recursively on $p$ by

$$
\gamma_{t k l}^{(L)}= \begin{cases}g_{L}^{\prime}\left(\overline{y_{t l}^{n e t}}\right) g_{L}^{\prime}\left(y_{t l}^{n e t}\right) & \text { if } k=l, \\ 0 & \text { if } k \neq l,\end{cases}
$$

for $k, l=1, \ldots, C$, and for $p=1, \ldots, L-1$,

$$
\gamma_{t j b}^{(p)}=\left[\sum_{\eta=1}^{K_{p+1}} \sum_{\beta=1}^{K_{p+1}} \gamma_{t \eta \beta}^{(p+1)} \overline{w_{\eta j}^{(p)}} w_{\beta b}^{(p)}\right] g_{p}^{\prime}\left(\overline{\left(x_{t j}^{(p)}\right)^{n e t}}\right) g_{p}^{\prime}\left(\left(x_{t b}^{(p)}\right)^{n e t}\right)
$$

for $j, b=1, \ldots, K_{p+1}$,

(2) the entries of the Hessian matrices $\mathcal{H}_{\overline{\mathbf{w}}^{(p-1)} \mathbf{w}^{(p-1)}}$ for $p=1, \ldots, L$ are given by

$$
\frac{\partial}{\partial \overline{w_{b a}^{(p-1)}}}\left(\frac{\partial E}{\partial w_{j i}^{(p-1)}}\right)^{*}=\frac{1}{N} \sum_{t=1}^{N}\left(\psi_{t j b}^{(p)}+\theta_{t j b}^{(p)}\right) \overline{x_{t i}^{(p-1)} x_{t a}^{(p-1)}}
$$

for $j, b=1, \ldots, K_{p}$ and $i, a=1, \ldots, K_{p-1}$, where the $\theta_{t j b}^{(p)}$ are defined for $t=1, \ldots, N$ by

$$
\theta_{t k l}^{(L)}= \begin{cases}\left(y_{t l}-d_{t l}\right) g_{L}^{\prime \prime}\left(\overline{y_{t l}^{n e t}}\right) & \text { if } k=l \\ 0 & \text { if } k \neq l\end{cases}
$$


for $k, l=1, \ldots, C$, and for $p=1, \ldots, L-1$,

$$
\theta_{t j b}^{(p)}= \begin{cases}{\left[\sum_{\eta=1}^{K_{p+1}} E_{t \eta}^{(p+1)} w_{\eta j}^{(p)}\right] g_{p}^{\prime \prime}\left(\overline{\left.\left(x_{t j}^{(p)}\right)^{n e t}\right)}\right.} & \text { if } j=b, \\ 0 & \text { if } j \neq b,\end{cases}
$$

for $j, b=1, \ldots, K_{p+1}$, where the $E_{t \eta}^{(p)}$ are given by 3.4) and 3.5), and the $\psi_{t j b}^{(p)}$ are defined for $t=1, \ldots, N$ recursively on $p$ by $\psi_{t k l}^{(L)}=0$ for $k, l=1, \ldots, C$, and for $p=1, \ldots, L-1$,

$$
\begin{aligned}
\psi_{t j b}^{(p)}=\left[\sum _ { \eta = 1 } ^ { K _ { p + 1 } } \sum _ { \beta = 1 } ^ { K _ { p + 1 } } \left(\psi_{t \eta \beta}^{(p+1)} \overline{w_{\beta b}^{(p)}}\right.\right. & \left.\left.+\theta_{t \eta \beta}^{(p+1)} \overline{w_{\eta b}^{(p)}}\right) \overline{w_{\eta j}^{(p)}}\right] \\
& \cdot g_{p}^{\prime}\left(\overline{\left(x_{t j}^{(p)}\right)^{n e t}}\right) g_{p}^{\prime}\left(\overline{\left(x_{t b}^{(p)}\right)^{n e t}}\right)
\end{aligned}
$$

for $j, b=1, \ldots, K_{p+1}$, and

(3) for the other two Hessian matrices we have $\mathcal{H}_{\mathbf{w}^{(p-1)}} \overline{\mathbf{w}^{(p-1)}}=$ $\overline{\mathcal{H}} \overline{\mathbf{w}^{(p-1)} \mathbf{w}^{(p-1)}}$ and $\mathcal{H} \overline{\mathbf{w}^{(p-1)} \mathbf{w}^{(p-1)}}=\overline{\mathcal{H}_{\mathbf{w}^{(p-1)} \mathbf{w}^{(p-1)}}}$.

Proof. (1) Setting $\gamma_{t k l}^{(L)}$ as defined above, Equation 4.13 follows immediately from (4.4). For the hidden layer Hessian matrix entries, set

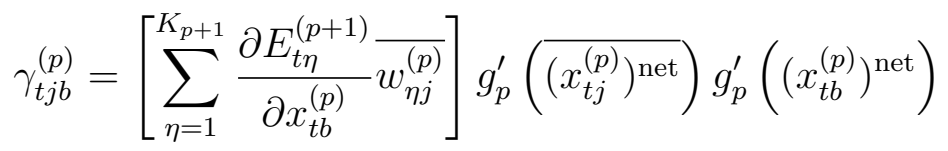

in (4.7), giving us 4.13). Then using 4.8 we have

$$
\frac{\partial E_{t \eta}^{(p+1)}}{\partial x_{t b}^{(p)}}=\sum_{\beta=1}^{K_{p+1}} \gamma_{t \eta \beta}^{(p+1)} w_{\beta b}^{(p)}
$$

So substituting (4.19) into 4.18) we get the recursive formula (4.14).

(2) The formula (4.15) for $p=L$ follows directly from the way we defined $\theta_{t k l}^{(L)}, \psi_{t k l}^{(L)}$, and equation 4.9 . Next, define the $\theta_{t j b}^{(p)}$ as above, and set

$$
\psi_{t j b}^{(p)}=\left[\sum_{\eta=1}^{K_{p+1}} \frac{\partial E_{t \eta}^{(p+1)}}{\partial \overline{x_{t b}^{(p)}}} \overline{w_{\eta j}^{(p)}}\right] g_{p}^{\prime}\left(\overline{\left(x_{t j}^{(p)}\right)^{\mathrm{net}}}\right) g_{p}^{\prime}\left(\overline{\left(x_{t b}^{(p)}\right)^{\mathrm{net}}}\right)
$$

in 4.10). Substituting 4.20 and 4.16 into 4.10 gives us 4.15). For the $\psi_{t j b}^{(p)}$, using 4.11 with our definition of the $\psi_{t j b}^{(p)}$ 
in 4.20 we have:

$$
\frac{\partial E_{t \eta}^{(p+1)}}{\partial \overline{x_{t b}^{(p)}}}=\sum_{\beta=1}^{K_{p+1}}\left(\psi_{t \eta \beta}^{(p+1)} \overline{w_{\beta b}^{(p)}}+\theta_{t \eta \beta}^{(p+1)} \overline{w_{\eta b}^{(p)}}\right)
$$

so substituting (4.21) into 4.20 we get 4.17).

\section{Backpropagation Using the Pseudo-Newton's Method}

To simplify the computation in the implementation of Newton's method, we can use the pseudo-Newton algorithm, which is an alternative algorithm also known to provide good quadratic convergence. For the pseudo-Newton algorithm, we take $\mathcal{H}_{\mathbf{w}^{(p-1)}} \mathbf{w}^{(p-1)}=0=\mathcal{H}_{\mathbf{w}^{(p-1)}} \overline{\mathbf{w}^{(p-1)}}$ in 4.12 , thus reducing the weight updates to

$$
\Delta \mathbf{w}^{(p-1)}=-\mathcal{H}_{\mathbf{w}^{(p-1)} \mathbf{w}^{(p-1)}}^{-1}\left(\frac{\partial E}{\partial \mathbf{w}^{(p-1)}}\right)^{*} .
$$

Convergence using the pseudo-Newton algorithm will generally be faster than gradient descent. The trade off for computational efficiency over Newton's method is somewhat slower convergence, though if the activation functions in the holomorphic MLP are in addition onto, the performance of the pseudo-Newton versus Newton algorithms should be similar 30.

Corollary 5.1 (Pseudo-Newton Backpropagation Algorithm for Holomorphic Neural Networks). The weight updates for the holomorphic $M L P$ with activation functions satisfying

$$
\overline{g(z)}=g(\bar{z})
$$

$p=1, \ldots, L$, using the backpropagation algorithm with the pseudoNewton's method are given by

$$
\Delta \mathbf{w}^{(p-1)}=-\mathcal{H}_{\mathbf{w}^{(p-1)} \mathbf{w}^{(p-1)}}^{-1}\left(\frac{\partial E}{\partial \mathbf{w}^{(p-1)}}\right)^{*},
$$

where the entries of the Hessian matrices $\mathcal{H}_{\mathbf{w}^{(p-1)} \mathbf{w}^{(p-1)}}$ for $1 \leq p \leq L$ are given by (4.13) in Theorem 4.1.

\section{The One-Step Newton Steplength Algorithm for Real-Valued Complex Functions}

A significant problem encountered with Newton's method and other minimization algorithms is the tendency of the iterates to "overshoot." If this happens, the iterates may not decrease the function value at each step [31]. For functions on real domains, it is known that for any 
minimization algorithm, careful choice of the sequence of steplengths via various steplength algorithms will guarantee a descent method. Steplength algorithms for minimization of real-valued functions on complex domains have been discussed in the literature [32, 12, 33, 34]. In [33], the problem was addressed by imposing unitary conditions on the input vectors. In [34, steplength algorithms were proposed for the BFGS method, which is an approximation to Newton's method. With regard to applications in neural networks, variable steplength algorithms exist for least mean square error algorithms, and these algorithms have been adapted to the gradient descent backpropagation algorithm for fully complex-valued neural networks with analytic activation functions [32, 27]. Fully adaptive gradient descent algorithms for complex-valued neural networks have also been proposed [12]. However, these algorithms do not apply to the Newton backpropagation algorithm.

To provide a steplength algorithm that guarantees convergence of Newton's method for real-valued complex functions, we need the following definitions. Let $f: \Omega \subseteq \mathbb{C}^{k} \rightarrow \mathbb{R}$. The function $f$ is called real differentiable ( $\mathbb{R}$-differentiable) if it is (Frechet) differentiable as a mapping

$$
f(\mathbf{x}, \mathbf{y}): D:=\left\{\left(\begin{array}{l}
\mathbf{x} \\
\mathbf{y}
\end{array}\right) \in \mathbb{R}^{2 k} \mid \begin{array}{c}
\mathbf{x}, \mathbf{y} \in \mathbb{R}^{k} \\
\mathbf{z}=\mathbf{x}+i \mathbf{y} \in \Omega
\end{array}\right\} \mathbb{R}^{2 k} \rightarrow \mathbb{R} .
$$

We then define a stationary point of $f$ to be a stationary point in the sense of the function $f(\mathbf{x}, \mathbf{y}): D \subseteq \mathbb{R}^{2 k} \rightarrow \mathbb{R}$. If $f$ is twice $\mathbb{R}$ differentiable, let $\mathcal{H}_{\mathbf{z z}}$ and $\mathcal{H}_{\overline{\mathbf{z}} \mathbf{z}}$ denote the Hessian matrices of $f$ with respect to $\mathbf{z}$ given by $(4.2)$.

Let $\mathbf{z}(0) \in \Omega$. If $\Omega$ is open, we define the level set of $\mathbf{z}(0)$ under $f$ on $\Omega$ to be

$$
L_{\mathbb{C}^{k}}(f(\mathbf{z}(0)))=\{\mathbf{z} \in \Omega \mid f(\mathbf{z}) \leq f(\mathbf{z}(0))\},
$$

and let $L_{\mathbb{C}^{k}}^{0}(f(\mathbf{z}(0)))$ be the path-connected component of $L_{\mathbb{C}^{k}}(f(\mathbf{z}(0)))$ containing $\mathbf{z}(0)$. To discuss rate of convergence, recall that the rootconvergence factors (R-factors) of a sequence $\{\mathbf{z}(n)\} \subseteq \mathbb{C}^{k}$ that converges to $\hat{\mathbf{z}} \in \mathbb{C}^{k}$ are

$$
R_{p}\{\mathbf{z}(n)\}= \begin{cases}\limsup \sup _{n \rightarrow \infty}\|\mathbf{z}(n)-\hat{\mathbf{z}}\|_{\mathbb{C}^{k}}^{1 / n} & \text { if } p=1 \\ \limsup _{n \rightarrow \infty}\|\mathbf{z}(n)-\hat{\mathbf{z}}\|_{\mathbb{C}^{k}}^{1 / p^{n}} & \text { if } p>1\end{cases}
$$

and the sequence is said to have at least an $\mathrm{R}$-linear rate of convergence if $R_{1}\{\mathbf{z}(n)\}<1$. 
The following theorem gives the one-step Newton steplength algorithm to adjust the sequence of steplengths for minimization of a realvalued complex function using Newton's method. We provide the detailed proof in Appendix B.

Theorem 6.1 (Convergence of the Complex Newton Algorithm with Complex One-Step Newton Steplengths). Let $f: \Omega \subseteq \mathbb{C}^{k} \rightarrow \mathbb{R}$ be twicecontinuously $\mathbb{R}$-differentiable on the open convex set $\Omega$ and assume that $L_{\mathbb{C}^{k}}^{0}(f(\mathbf{z}(0)))$ is compact for $\mathbf{z}(0) \in \Omega$. Suppose for all $\mathbf{z} \in \Omega$,

$$
\operatorname{Re}\left\{\mathbf{h}^{*} \mathcal{H}_{\mathbf{z z}}(\mathbf{z}) \mathbf{h}+\mathbf{h}^{*} \mathcal{H}_{\overline{\mathbf{z}} \mathbf{z}}(\mathbf{z}) \overline{\mathbf{h}}\right\}>0 \text { for all } \mathbf{h} \in \mathbb{C}^{k} .
$$

Assume $f$ has a unique stationary point $\hat{\mathbf{z}} \in L_{\mathbb{C}^{k}}^{0}(f(\mathbf{z}(0)))$, and fix $\epsilon \in(0,1]$. Consider the iteration

$$
\mathbf{z}(n+1)=\mathbf{z}(n)-\omega(n) \mu(n) \mathbf{p}(n), n=0,1, \ldots,
$$

where the $\mathbf{p}(n)$ are the nonzero complex Newton updates

$$
\begin{aligned}
\mathbf{p}(\mathbf{z}(n))= & -\left[\mathcal{H}_{\mathbf{z z}}(\mathbf{z}(n))-\mathcal{H}_{\overline{\mathbf{z}} \mathbf{z}}(\mathbf{z}(n)) \mathcal{H}_{\overline{\mathbf{z}}}(\mathbf{z}(n))^{-1} \mathcal{H}_{\mathbf{z} \overline{\mathbf{z}}}(\mathbf{z}(n))\right]^{-1} \\
& \cdot\left[\mathcal{H}_{\overline{\mathbf{z}} \mathbf{z}}(\mathbf{z}(n)) \mathcal{H}_{\overline{\mathbf{z z}}}(\mathbf{z}(n))^{-1}\left(\frac{\partial f}{\partial \overline{\mathbf{z}}}(\mathbf{z}(n))\right)^{*}-\left(\frac{\partial f}{\partial \mathbf{z}}(\mathbf{z}(n))\right)^{*}\right],
\end{aligned}
$$

the steplengths $\mu(n)$ are given by

$$
\mu(n)=\frac{\operatorname{Re}\left\{\frac{\partial f}{\partial \mathbf{z}}(\mathbf{z}(n)) \mathbf{p}(n)\right\}}{\operatorname{Re}\left\{\mathbf{p}(n) * \mathcal{H}_{\mathbf{z z}}(\mathbf{z}(n)) \mathbf{p}(n)+\mathbf{p}(n) * \mathcal{H}_{\overline{\mathbf{z} z}}(\mathbf{z}(n)) \overline{\mathbf{p}(n)}\right\}},
$$

and the underrelaxation factors $\omega(n)$ satisfy

$$
0 \leq \epsilon \leq \omega(n) \leq \frac{2}{\gamma(n)}-\epsilon
$$

where, taking $\mathbf{z}=\mathbf{z}(n)$ and $\mathbf{p}=\mathbf{p}(n)$,

$$
\gamma(n)=\sup \left\{\begin{array}{c}
\frac{\operatorname{Re}\left\{\mathbf{p}^{*} \mathcal{H}_{\mathbf{z z}}(\mathbf{z}-\mu \mathbf{p}) \mathbf{p}+\mathbf{p}^{*} \mathcal{H}_{\overline{\mathbf{z}} \mathbf{z}}(\mathbf{z}-\mu \mathbf{p}) \overline{\mathbf{p}}\right\}}{\operatorname{Re}\left\{\mathbf{p}^{*} \mathcal{H}_{\mathbf{z z}}(\mathbf{z}) \mathbf{p}+\mathbf{p}^{*} \mathcal{H}_{\overline{\mathbf{z}} \mathbf{z}}(\mathbf{z}) \overline{\mathbf{p}}\right\}} \mid \\
\mu>0, f(\mathbf{z}-\nu \mathbf{p})<f(\mathbf{z}) \\
\text { for all } \nu \in(0, \mu]
\end{array}\right\} .
$$

Then $\lim _{n \rightarrow \infty} \mathbf{z}(n)=\hat{\mathbf{z}}$, and the rate of convergence is at least $R$-linear.

To apply the one-step Newton steplength algorithm to the Newton's method or pseudo-Newton's method backpropagation algorithm 
for complex-valued holomorphic multilayer perceptrons, at the $n$th iteration in the training process, the one-step Newton steplength for the $p$ th step in the backpropagation $(1 \leq p \leq L)$ is

$$
\mu_{p}(n)=\frac{-\operatorname{Re}\left(\frac{\partial E}{\partial \mathbf{w}} \Delta \mathbf{w}\right)}{\operatorname{Re}\left\{(\Delta \mathbf{w})^{*} \mathcal{H}_{\mathbf{w w}} \Delta \mathbf{w}+(\Delta \mathbf{w})^{*} \mathcal{H}_{\overline{\mathbf{w}} \mathbf{w}} \overline{\Delta \mathbf{w}}\right\}},
$$

where $\Delta \mathbf{w}=\Delta \mathbf{w}^{(p-1)}$ is the weight update for the $p$ th layer of the network given by Theorem 4.1 or Corollary 5.1, respectively, and $\mathbf{w}=$ $\mathbf{w}^{(p-1)}$. (Recall (3.2), so that here $\mathbf{p}(n)=-\Delta \mathbf{w}^{(p-1)}$ in (6.3).) For the pseudo-Newton's method backpropagation, we set $\mathcal{H}_{\mathbf{w}^{(p-1)}} \mathbf{w}^{(p-1)}=$ $\mathcal{H}_{\mathbf{w}^{(p-1)}} \overline{\mathbf{w}^{(p-1)}}=0$ in 6.4 to obtain the pseudo-Newton updates $\Delta \mathbf{w}^{(p-1)}$ given in Corollary 5.1. but leave $\mathcal{H}_{\mathbf{w}^{(p-1)}} \mathbf{w}^{(p-1)}$ as calculated in Theorem 4.1 in 6.7). In theory, for the $n$th iteration in the training process, we should choose the underrelaxation factor $\omega_{p}(n)$ for the $p$ th step in the backpropagation $(1 \leq p \leq L)$ according to (6.5) and (6.6). However, in practical application it suffices to take the underrelaxation factors to be constant and they may be chosen experimentally to yield convergence of the error function (see our results in Section VII). It is also not necessary in practical application to verify all the conditions of Theorem 6.1. In particular we may assume that the error function has a stationary point sufficiently close to the initial weights since the initial weights were chosen specifically to be "nearby" a stationary point, and that the stationary point is unique in the appropriate compact level set of the initial weights since the set of zeros of the error function has measure zero.

\section{EXPERIMENTS}

To test the efficiency of the algorithms in the previous sections, we will compare the results of applying the gradient descent method, Newton's method, and the pseudo-Newton's method to a holomorphic MLP trained with data from the real-valued exclusive-or (XOR) problem (see

\begin{tabular}{|c|c|}
\hline Input Pattern & Output \\
\hline 00 & 0 \\
\hline 10 & 1 \\
\hline 01 & 1 \\
\hline 11 & 0 \\
\hline
\end{tabular}

TABle 1. XOR Training Set 

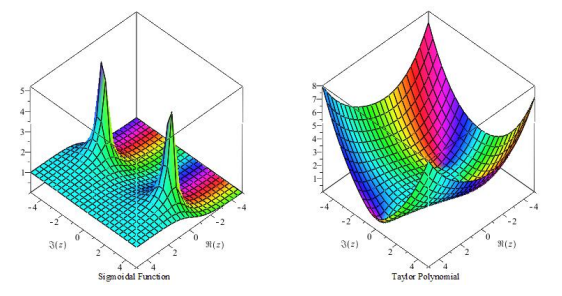

Figure 2. The sigmoidal function (left) has two poles in a region near 0 , while a Taylor polynomial approximation (right) of the sigmoidal function is bounded on the same region.

Table 1). Note that the complex-valued XOR problem has different criteria for the data set [18]. We use the real-valued XOR problem as we desire a complex-valued network to process real as well as complex data.

The XOR problem is frequently encountered in the literature as a test case for backpropagation algorithms [8]. A multilayer network is required to solve it: without hidden units the network is unable to distinguish overlapping input patterns which map to different output patterns, e.g. $(0,0)$ and $(1,0)$ [35]. We use a two-layer network with $m=2$ input nodes, $K=4$ hidden nodes, and $C=1$ output nodes. Any Boolean function of $m$ variables can be trained to a two-layered real-valued neural network with $2^{m}$ hidden units. Modeling after the real case we choose $K=2^{m}$, although this could perhaps be accomplished with fewer hidden units, as $2^{m-1}$ is a smaller upper bound for real-valued neural networks [36]. Some discussion of approximating Boolean functions, including the XOR and parity problems, using complex-valued neural networks is given in [37.

In our experiments, the activation functions are taken to be the same for both the hidden and output layers of the network. The activation function is either the sigmoidal function or its third degree $e^{1}$ Taylor polynomial approximation

$$
g(z)=\frac{1}{1+\exp (-z)} \text { or } T(z)=\frac{1}{2}+\frac{1}{4} z-\frac{1}{48} z^{3} .
$$

Notice that while $g(z)$ has poles near zero, the polynomial $T(z)$ is analytic on the entire complex plane and bounded on bounded regions (see Figure 2).

\footnotetext{
${ }^{1}$ One can take a higher degree Taylor polynomial approximation, but this is sufficient for our purposes.
} 
For each activation function we trained the network using the gradient descent backpropagation algorithm, the Newton backpropagation algorithm, and the pseudo-Newton backpropagation algorithm. The real and imaginary parts of the initial weights for each trial were chosen randomly from the interval $[-1,1]$ according to a uniform distribution. In each case the network was trained to within 0.001 error. One hundred trials were performed for each activation function and each backpropagation algorithm (note that the same set of random initial weights was used for each set of trials). For the trials using the gradient descent backpropagation algorithm, a constant learning rate $(\mu)$ was used. It is known that for the gradient descent algorithm for real-valued neural networks, some learning rates will result in nonconvergence of the error function [38. There is experimental evidence that for elementary transcendental activation functions used in complex-valued neural networks, sensitivity of the gradient descent algorithm to the choice of the learning rate can result in nonconvergence of the error function as well, and this is not necessarily affected by changes in the initial weight distribution [18]. To avoid these problems, a learning rate of $\mu=1$ was chosen both to guarantee convergence and to yield fast convergence (as compared to other values of $\mu$ ). For the trials using the Newton and pseudo-Newton backpropagation algorithms, a variable learning rate (steplength) was chosen according to the one-step Newton steplength algorithm (Theorem 6.1) to control the problem of "overshooting" of the iterates and nonconvergence of the error function when a fixed learning rate was used. For both the Newton and pseudo-Newton trials, a constant underrelaxation factor of $\omega=0.5$ was used; this was chosen to yield the best chance for convergence of the error function. The results are summarized in Table 2.

Over the successful trials, the polynomial activation function performed just as well as the traditional sigmoidal function for the gradient descent backpropagation algorithm and yielded more successful trials than the sigmoidal function for the Newton and pseudo-Newton backpropagation algorithms. We define a successful trial to be one in which the error function dropped below 0.001. We logged four different types of unsuccessful trials (see Table 3). Convergence of the error function to a local minimum occurred when, after at least 50,000 iterations for gradient descent and 5,000 iterations for the Newton and pseudo-Newton algorithms, the error function remained above 0.001 but had stabilized to within $10^{-10}$ between successive iterations. This occurred more frequently in the Newton's method trails than the gradient descent trials, which was expected due to the known sensitivity 


\begin{tabular}{|c|c|c|c|c|c|}
\hline $\begin{array}{c}\text { Activation } \\
\text { Function }\end{array}$ & $\begin{array}{c}\text { Training } \\
\text { Method }\end{array}$ & $\begin{array}{c}\text { Learning } \\
\text { Rate }(\mu)\end{array}$ & $\begin{array}{c}\text { Underrelaxation } \\
\text { Factor }(\omega)\end{array}$ & $\begin{array}{c}\text { Number of } \\
\text { Successful } \\
\text { Trials }\end{array}$ & $\begin{array}{c}\text { Average } \\
\text { Number of } \\
\text { Iterations* }\end{array}$ \\
\hline Sigmoidal & $\begin{array}{c}\text { Gradient } \\
\text { Descent }\end{array}$ & $\mu=1$ & None & 93 & 1258.9 \\
\hline Sigmoidal & Newton & $\begin{array}{c}\text { One-Step } \\
\text { Newton }\end{array}$ & $\omega=0.5$ & 5 & 7.0 \\
\hline Sigmoidal & $\begin{array}{c}\text { Pseudo- } \\
\text { Newton }\end{array}$ & $\begin{array}{c}\text { One-Step } \\
\text { Newton }\end{array}$ & $\omega=0.5$ & 78 & 7.0 \\
\hline Polynomial & $\begin{array}{c}\text { Gradient } \\
\text { Descent }\end{array}$ & $\mu=1$ & None & 93 & 932.2 \\
\hline Polynomial & Newton & $\begin{array}{c}\text { One-Step } \\
\text { Newton }\end{array}$ & $\omega=0.5$ & 53 & 107.9 \\
\hline Polynomial & $\begin{array}{c}\text { Pseudo- } \\
\text { Newton }\end{array}$ & $\begin{array}{c}\text { One-Step } \\
\text { Newton }\end{array}$ & $\omega=0.5$ & 99 & 23.7 \\
\hline
\end{tabular}

*Over the successful trials.

TABLE 2. XOR Experiment Results

\begin{tabular}{|c|c|c|c|c|c|c|}
\hline $\begin{array}{c}\text { Activation } \\
\text { Function }\end{array}$ & $\begin{array}{c}\text { Training } \\
\text { Method }\end{array}$ & $\begin{array}{c}\text { Local } \\
\text { Minimum }\end{array}$ & $\begin{array}{c}\text { Blow } \\
\text { Up }\end{array}$ & $\begin{array}{c}\text { Undefined } \\
\text { Floating } \\
\text { Point }\end{array}$ & $\begin{array}{c}\text { Singular } \\
\text { Matrix }\end{array}$ & $\begin{array}{c}\text { Total } \\
\text { Unsuccessful } \\
\text { Trials }\end{array}$ \\
\hline Sigmoidal & $\begin{array}{c}\text { Gradient } \\
\text { Descent }\end{array}$ & 1 & 0 & 6 & N/A & 7 \\
\hline Sigmoidal & Newton & 0 & 0 & 68 & 27 & 95 \\
\hline Sigmoidal & $\begin{array}{c}\text { Pseudo- } \\
\text { Newton }\end{array}$ & 0 & 0 & 14 & 8 & 22 \\
\hline Polynomial & $\begin{array}{c}\text { Gradient } \\
\text { Descent }\end{array}$ & 0 & 0 & 7 & N/A & 7 \\
\hline Polynomial & Newton & 26 & 2 & 2 & 17 & 47 \\
\hline Polynomial & $\begin{array}{c}\text { Pseudo- } \\
\text { Newton }\end{array}$ & 1 & 0 & 0 & 0 & 1 \\
\hline
\end{tabular}

TABLE 3. Unsuccessful Trials

of Newton's method to the initial points. A blow up of the error function occurred when, after the same minimum number of iterations as above, the error function had increased to above $10^{10}$. The final value of the error function was sometimes an undefined floating point number, probably the result of division by zero. This occurred less frequently with the polynomial activation function than with the sigmoidal activation function. Finally, the last type of unsuccessful trial resulted from a singular Hessian matrix (occurring only in the Newton and pseudoNewton trials). This, necessarily, halted the backpropagation process, and occurred less frequently with the polynomial activation function than with the sigmoidal activation function.

As for efficiency, the Newton and pseudo-Newton algorithms required significantly fewer iterations of the backpropagation algorithm to train 
the network than the gradient descent method for each activation function. In addition to producing fewer unsuccessful trials, the pseudoNewton algorithm yielded a lower average number of iterations than the Newton algorithm for the polynomial activation function and the same average number of iterations as the Newton algorithm for the sigmoidal activation function. The network with polynomial activation function trained using the pseudo-Newton algorithm produced the fewest unsuccessful trials. Overall, we conclude that the use of the polynomial activation function yields more consistent convergence of the error function than the use of the sigmoidal activation function, and the use of the Newton and pseudo-Newton algorithms yields significantly fewer training iterations than the use of the gradient descent method.

\section{Conclusion}

We have developed the backpropagation algorithm using Newton's method for complex-valued holomorphic multilayer perceptrons. The extension of real-valued neural networks to complex-valued neural networks is natural and doing so allows the proper treatment of the phase information. However, the choice of nonlinear activation functions poses a challenge in the backpropagation algorithm. The usual complex counterparts of the commonly used real-valued activation functions are no longer unbounded: they have poles near zero, while other choices are not fully complex-valued functions. To provide experimental evidence for the choice of holomophic functions as activation functions in addition to mathematical reasoning, we compared the results of using the complex-valued sigmoidal function as activation functions and the results of using its Taylor polynomial approximation as activation functions. Our experiments showed that when Newton's method was used for the XOR example, Taylor polynomial approximations are better choices. The use of polynomials as activation functions allows the possibility of rigorous analysis of performance of the algorithm, as well as making connections with other topics of complex analysis, which are virtually nonexistent in complex-valued neural network studies so far. These topics are under investigation currently.

\section{REFERENCES}

[1] Akira Hirose. Complex-Valued Neural Networks, volume 400 of Studies in Computational Intelligence. Springer-Verlag Berlin Heidelberg, New York, 2nd edition, 2012.

[2] Akira Hirose. Nature of complex number and complex-valued neural networks. Frontiers of Electrical and Electronic Engineering in China, 6(1):171-180, 2011. 
[3] Indrajit Mukherjee and Srikanta Routroy. Comparing the performance of neural networks developed by using levenbergmarquardt and quasi-newton with the gradient descent algorithm for modelling a multiple response grinding process. Expert Systems with Applications, 39:2397-2407, February 2012.

[4] John B. Conway. Functions of One Complex Variable I. Graduate Texts in Mathematics. Springer Science+Business Media, Inc., New York, 2 edition, 1978.

[5] George M. Georgiou and Cris Koutsougeras. Complex domain backpropagation. IEEE Transactions on Circuits and Systems-II: Analog and Digital Signal Processing, 39(5):300-334, May 1992.

[6] Hamid A. Jalab and Rabha W. Ibrahim. New activation functions for complex-valued neural network. International Journal of the Physical Sciences, 6(7):1766-1772, April 2011.

[7] Taehwan Kim and Tülay Adali. Approximation by fully complex mlp using elementary transcendental functions. In Neural Networks for Signal Processing XI, 2001. Proceedings of the 2001 IEEE Signal Processing Society Workshop, pages 203-212. IEEE, 2001.

[8] Anupama Pande and Vishik Goel. Complex-valued neural network in image recognition: A study on the effectiveness of radial basis function. World Academy of Science, Engineering and Technology, 26:220-225, 2007.

[9] Md. Faijul Amin and Kazuyuki Murase. Single-layered complex-valued neural network for real-valued classification problems. Neurocomputing, 72:945-955, 2009.

[10] Md. Faijul Amin, Md. Monirul Islam, and Kazuyuki Murase. Ensemble of single-layered complex-valued neural networks for classification tasks. Neurocomputing, 72:2227-2234, 2009.

[11] Md. Faijul Amin, Md. Monirul Islam, and Kazuyuki Murase. Single-layered complex-valued neural networks and their ensembles for real-valued classification problems. In 2008 International Joint Conference on Neural Networks, pages 2500-2506. IEEE, 2008.

[12] Andrew Ian Hanna and Danilo P. Mandic. A fully adaptive normalized nonlinear gradient descent algorithm for complex-valued nonlinear adaptive filters. IEEE Transactions on Signal Processing, 51(10):2540-2549, October 2003.

[13] Taehwan Kim and Tülay Adali. Fully complex multi-layer perceptron network for nonlinear signal processing. Journal of VLSI Signal Processing Systems, 32(1/2):29-43, August-September 2002.

[14] Kavita Burse, Anjana Pandey, and Ajay Somkuwar. Convergence analysis of complex valued multiplicative neural network for various activation functions. In 2011 International Conference on Computational Intelligence and Communication Systems, pages 279-282, 2011.

[15] Ming-Bin Li, Guang-Bin Huang, P. Saratchandran, and N. Sundararajan. Fully complex extreme learning machine. Neurocomputing, 68:306-314, October 2005 .

[16] R. Savitha, S. Suresh, N. Sundararajan, and H.J. Kim. Fast learning fully complex-valued classifiers for real-valued classification problems. In D. Liu et al, editor, Advances in Neural Networks-ISNN 2011, Part I, volume 6675 of Lecture Notes in Computer Science, pages 602-609. Springer-Verlag Berlin Heidelberg, 2011. 
[17] Henry Leung and Simon Haykin. The complex backpropagation algorithm. IEEE Transactions on Signal Processing, 39(9):2101-2104, September 1991.

[18] R. Savitha, S. Suresh, N. Sundararajan, and P. Saratchandran. A new learning algorithm with logarithmic performance index for complex-valued neural networks. Neurocomputing, 72:3771-3781, 2009.

[19] Md. Faijul Amin, Ramasamy Savitha, Muhammad Ilias Amin, and Kazuyuki Murase. Complex-valued functional link network design by orthogonal least squares method for function approximation problems. In Proceedings of the International Joint Conference on Neural Networks, pages 1489-1496, July/August 2011.

[20] Md. Faijul Amin, Ramasamy Savitha, Muhammad Ilias Amin, and Kazuyuki Murase. Orthogonal least squares based complex-valued functional link network. Neural Networks, 32:257-266, 2012. 2012 Special Issue.

[21] Michael Reed and Barry Simon. Methods of Modern Mathematical Physics I: Functional Analysis. Academic Press, London, 1980.

[22] Sven Buchholz and Gerald Sommer. On clifford neurons and clifford multi-layer perceptrons. Neural Networks, 21:925-935, 2008.

[23] M.S. Al-Haik, H. Garmestani, and I.M. Navon. Truncated-newton training algorithm for neurocomputational viscoplastic model. Computational methods in applied mechanics and engineering, 192:2249-2267, 2003.

[24] H.S.M. Beigi and C.J. Li. Learning algorithms for neural networks based on quasi-newton methods with self-scaling. Journal of Dynamical Systems, Measurement, and Control, 115:38-43, March 1993.

[25] Martin T. Hagan and Mohammad B. Menhaj. Training feedforward networks with the marquardt algorithm. IEEE Transactions on Neural Networks, 5(6):989-993, November 1994.

[26] Hao Yu and Bogdan M. Wilamowski. Levenberg-marquardt training. In Industrial Electronics Handbook, Vol. 5: Intelligent Systems, chapter 12, pages 12-1 - 12-15. CRC Press, 2 edition, 2011.

[27] Su Lee Goh and Danilo P. Mandic. A class of gradient-adaptive step size algorithms for complex-valued nonlinear neural adaptive filters. In IEEE International Conference on Acoustics, Speech, and Signal Processing, 2005. Proceedings. (ICASSP '05), volume 5, pages V/253-V/256. IEEE, May 2005.

[28] Hans Georg Zimmermann, Alexey Minin, and Victoria Kusherbaeva. Comparison of the complex valued and ral valued neural networks trained with gradient descent and random search algorithms. In European Symposium on Artificial Neural Networks, Computational Intelligence and Machine Learning, pages 213-218, Bruges (Belgium), April 2011.

[29] Hualiang Li and Tülay Adali. Complex-valued adaptive signal processing using nonlinear functions. EURASIP Journal on Advances in Signal Processing, 2008, 2008.

[30] Ken Kreutz-Delgado. The complex gradient operator and the $\mathbb{C} \mathbb{R}$-calculus. University of California, San Diego, Version UCSD-ECE275CG-S2009v1.0, 25 June 2009. arXiv:0906.4835v1 [math.OC], June 2009.

[31] J.M. Ortega and W.C. Rheinboldt. Iterative Solution of Nonlinear Equations in Several Variables. Academic Press, Inc., New York, NY, 1970. 
[32] Wee-Peng Ang and B. Farhang-Boroujeny. A new class of gradient adaptive step-size lms algorithms. IEEE Transactions on Signal Processing, 49(4):805810, April 2001.

[33] Jonathan H. Manton. Optimization algorithms exploiting unitary constraints. IEEE Transactions on Signal Processing, 50(3):635-650, March 2002.

[34] Laurent Sorber, Marc Van Barel, and Lieven De Lathauwer. Unconstrained optimization of real functions in complex variables. Technical Report TW592, Katholieke Universiteit Leuven, Heverlee (Belgium), April 2011.

[35] D.E. Rumelhart, G.E. Hinton, and R.J. Williams. Learning internal representations by error propagation. In D.E. Rumelhart and J.L. McCelland, editors, Parallel Distributed Processing: Explorations in the Microstructure of Cognition, volume 1, chapter 8. Foundations M.I.T. Press, Cambridge, MA, 1986.

[36] Mohamad H. Hassoun. Fundamentals of Artificial Neural Networks. The MIT Press, Cambridge, MA, 1995.

[37] Iku Nemoto and Tomoshi Kono. Complex neural networks. Systems and Computers in Japan, 23(8):75-84, 1992. Translated from Denshi Joho Tsushin Gakkai Ronbunshi, Vol. 74-D-II, No. 9, pp. 1282-1288, September 1991.

[38] Yann Le Cun, Ido Kanter, and Sara A. Solla. Eigenvalues of covariance matrices: Application to neural-network learning. Physical Review Letters, 66(18):2396-2399, May 1991.

[39] Reinhold Remmert. Theory of Complex Functions. Springer-Verlag, New York, NY, 1991.

\section{Appendix A. Derivation of the Entries of the Hessian MATRICES FOR THE NeWTON'S METHOD BackPropagation Algorithm}

We give the detail, which was omitted in the main body of the paper, for the computation of the entries of the Hessian matrices $\mathcal{H}_{\overline{\mathrm{w} w}}$ for the $(p-1)$ th layer of the holomorphic multilayer perceptron, $1 \leq p \leq L$, which are given recursively by (4.10) and (4.11), in a manner similar to the computation of the Hessian matrices $\mathcal{H}_{w w}$ given in Section IV. Using the cogradients (3.3) we have:

$$
\overline{\partial \overline{w_{b a}^{(p-1)}}}\left(\frac{\partial E}{\partial w_{j i}^{(p-1)}}\right)^{*}=\frac{1}{N} \sum_{t=1}^{N} \frac{\partial E_{t j}^{(p)}}{\partial \overline{w_{b a}^{(p-1)}}} \overline{x_{t i}^{(p-1)}},
$$

where $j, b=1, \ldots, K_{p}$ and $i, a=1, \ldots, K_{p-1}$. Using (3.5),

$$
\begin{aligned}
& \frac{\partial E_{t j}^{(p)}}{\partial \overline{w_{b a}^{(p-1)}}}=\frac{\partial}{\partial \overline{w_{b a}^{(p-1)}}}\left[( \sum _ { \eta = 1 } ^ { K _ { p + 1 } } E _ { t \eta } ^ { ( p + 1 ) } \overline { w _ { \eta j } ^ { ( p ) } } ) g _ { p } ^ { \prime } \left(\overline{\left.\left.\left(x_{t j}^{(p)}\right)^{\mathrm{net}}\right)\right]}\right.\right. \\
& =g_{p}^{\prime}\left(\overline{\left(x_{t j}^{(p)}\right)^{\mathrm{net}}}\right) \sum_{\eta=1}^{K_{p+1}} \frac{\partial E_{t \eta}^{(p+1)}}{\partial \overline{w_{b a}^{(p-1)}}} \overline{w_{\eta j}^{(p)}}+\frac{\partial g_{p}^{\prime}\left(\overline{\left(x_{t j}^{(p)}\right)^{\mathrm{net}}}\right)}{\partial \overline{w_{b a}^{(p-1)}}} \sum_{\eta=1}^{K_{p+1}} E_{t \eta}^{(p+1)} \overline{w_{\eta j}^{(p)}},
\end{aligned}
$$


where

$$
\begin{aligned}
\frac{\partial E_{t \eta}^{(p+1)}}{\partial \overline{w_{b a}^{(p-1)}}} & =\frac{\partial E_{t \eta}^{(p+1)}}{\partial x_{t b}^{(p)}} \frac{\partial x_{t b}^{(p)}}{\partial \overline{w_{b a}^{(p-1)}}}+\frac{\partial E_{t \eta}^{(p+1)}}{\partial \overline{x_{t b}^{(p)}}} \frac{\overline{\partial x_{t b}^{(p)}}}{\partial \overline{w_{b a}^{(p-1)}}} \\
& =\frac{\partial E_{t \eta}^{(p+1)}}{\partial \overline{x_{t b}^{(p)}}}\left[\frac{\partial \overline{x_{t b}^{(p)}}}{\partial \overline{\left(x_{t b}^{(p)}\right)^{\text {net }}}} \frac{\partial \overline{\left(x_{t b}^{(p)}\right)^{\text {net }}}}{\partial \overline{w_{b a}^{(p-1)}}}+\frac{\partial \overline{x_{t b}^{(p)}}}{\partial\left(x_{t b}^{(p)}\right)^{\text {net }}} \frac{\partial\left(x_{t b}^{(p)}\right)^{\text {net }}}{\partial \overline{w_{b a}^{(p-1)}}}\right] \\
& =\frac{\partial E_{t \eta}^{(p+1)}}{\partial \overline{x_{t b}^{(p)}}} g_{p}^{\prime}\left(\overline{\left(x_{t b}^{(p)}\right)^{\text {net }}}\right) \overline{x_{t a}^{(p-1)}}
\end{aligned}
$$

and

$$
\begin{aligned}
& \frac{\partial g_{p}^{\prime}\left(\overline{\left(x_{t j}^{(p)}\right)^{\mathrm{net}}}\right)}{\partial \overline{w_{b a}^{(p-1)}}} \\
& =\frac{\partial g_{p}^{\prime}\left(\overline{\left(x_{t j}^{(p)}\right)^{\mathrm{net}}}\right)}{\partial \overline{\left(x_{t j}^{(p)}\right)^{\mathrm{net}}}} \frac{\overline{\left(x_{t j}^{(p)}\right)^{\mathrm{net}}}}{\partial \overline{w_{b a}^{(p-1)}}}+\frac{\partial g_{p}^{\prime}\left(\overline{\left(x_{t j}^{(p)}\right)^{\mathrm{net}}}\right)}{\partial\left(x_{t j}^{(p)}\right)^{\mathrm{net}}} \frac{\left(x_{t j}^{(p)}\right)^{\mathrm{net}}}{\partial \overline{w_{b a}^{(p-1)}}} \\
& = \begin{cases}g_{p}^{\prime \prime}\left(\overline{\left(x_{t j}^{(p)}\right)^{\mathrm{net}}}\right) \overline{x_{t a}^{(p-1)}} & \text { if } j=b, \\
0 & \text { if } j \neq b,\end{cases}
\end{aligned}
$$

so that

(A.2)

$$
\frac{\partial E_{t j}^{(p)}}{\partial \overline{w_{b a}^{(p-1)}}}=\left\{\begin{array}{c}
\left\{\left[\sum_{\eta=1}^{K_{p+1}} \frac{\partial E_{t \eta}^{(p+1)} \overline{x_{t b}^{(p)}}}{w_{\eta j}^{(p)}}\right] g_{p}^{\prime}\left(\overline{\left(x_{t j}^{(p)}\right)^{\mathrm{net}}}\right) g_{p}^{\prime}\left(\overline{\left(x_{t b}^{(p)}\right)^{\mathrm{net}}}\right)\right. \\
\left.+\left[\sum_{\eta=1}^{K_{p+1}} E_{t \eta}^{(p+1)} \overline{w_{\eta j}^{(p)}}\right] g_{p}^{\prime \prime}\left(\overline{\left(x_{t j}^{(p)}\right)^{\mathrm{net}}}\right)\right\} \overline{x_{t a}^{(p-1)}} \\
\text { if } j=b, \overline{\left(\sum_{\eta=1}^{K_{p+1}} \frac{\partial E_{t \eta}^{(p+1)}}{\partial x_{t b}^{(p)}} \overline{w_{\eta j}^{(p)}}\right]} g_{p}^{\prime}\left(\overline{\left.\left(x_{t j}^{(p)}\right)^{\text {net }}\right) g_{p}^{\prime}\left(\overline{\left(x_{t b}^{(p)}\right)^{\mathrm{net}}}\right) \overline{x_{t a}^{(p-1)}}}\right. \\
\text { if } j \neq b .
\end{array}\right.
$$


Combining (A.1) and (A.2), we get

$$
\begin{aligned}
& \frac{\partial}{\partial \overline{w_{b a}^{(p-1)}}}\left(\frac{\partial E}{\partial w_{j i}^{(p-1)}}\right)^{*}
\end{aligned}
$$

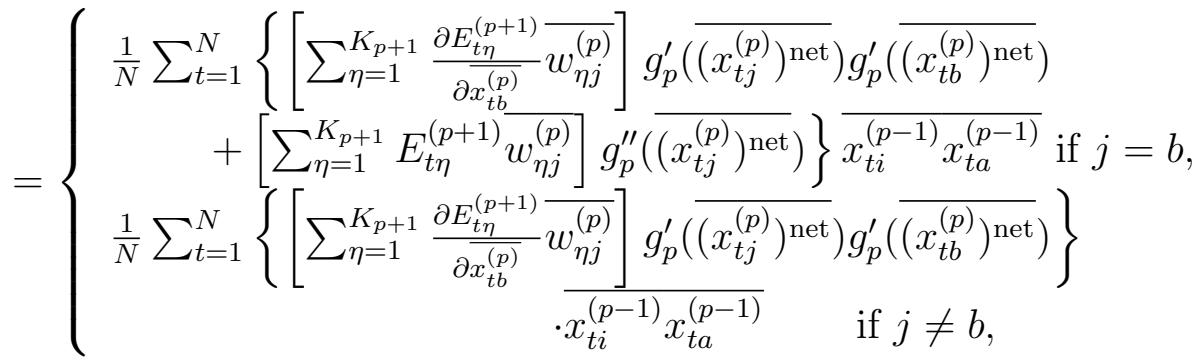

where $\frac{\partial E_{t \eta}^{(p+1)}}{\partial \overline{x_{t b}^{(p)}}}$ can be computed recursively:

$$
\begin{aligned}
& \frac{\partial E_{t \eta}^{(p+1)}}{\partial \overline{x_{t b}^{(p)}}}=\frac{\partial}{\partial \overline{x_{t b}^{(p)}}}\left[\left(\sum_{\alpha=1}^{K_{p+2}} E_{t \alpha}^{(p+2)} \overline{w_{\alpha \eta}^{(p+1)}}\right) g_{p+1}^{\prime}\left(\overline{\left(x_{t \eta}^{(p+1)}\right)^{\mathrm{net}}}\right)\right] \\
& =g_{p+1}^{\prime}\left(\overline{\left(x_{t \eta}^{(p+1)}\right)^{\mathrm{net}}}\right) \sum_{\alpha=1}^{K_{p+2}} \frac{\partial E_{t \alpha}^{(p+2)}}{\partial \overline{x_{t b}^{(p)}}} \overline{w_{\alpha \eta}^{(p+1)}} \\
& +\frac{\partial g_{p+1}^{\prime}\left(\overline{\left(x_{t \eta}^{(p+1)}\right)^{\mathrm{net}}}\right)}{\partial \overline{x_{t b}^{(p)}}} \sum_{\alpha=1}^{K_{p+2}} E_{t \alpha}^{(p+2)} \overline{w_{\alpha \eta}^{(p+1)}}
\end{aligned}
$$




$$
\begin{aligned}
& =g_{p+1}^{\prime}\left(\overline { ( x _ { t \eta } ^ { ( p + 1 ) } ) ^ { \mathrm { net } } ) } \sum _ { \alpha = 1 } ^ { K _ { p + 2 } } \sum _ { \beta = 1 } ^ { K _ { p + 1 } } \left[\frac{\partial E_{t \alpha}^{(p+2)}}{\partial x_{t \beta}^{(p+1)}} \frac{\partial x_{t \beta}^{(p+1)}}{\partial \overline{x_{t b}^{(p)}}}\right.\right. \\
& \left.+\frac{\partial E_{t \alpha}^{(p+2)}}{\partial \overline{x_{t \beta}^{(p+1)}}} \frac{\partial \overline{x_{t \beta}^{(p+1)}}}{\partial \overline{x_{t b}^{(p)}}}\right] \overline{w_{\alpha \eta}^{(p+1)}} \\
& +\left[\frac{\partial g_{p+1}^{\prime}\left(\overline{\left(x_{t \eta}^{(p+1)}\right)^{\mathrm{net}}}\right)}{\partial \overline{\left(x_{t \eta}^{(p+1)}\right)^{\mathrm{net}}}} \frac{\partial \overline{\left(x_{t \eta}^{(p+1)}\right)^{\mathrm{net}}}}{\partial \overline{x_{t b}^{(p)}}}\right. \\
& \left.+\frac{\partial g_{p+1}^{\prime}\left(\overline{\left(x_{t \eta}^{(p+1)}\right)^{\text {net }}}\right)}{\partial\left(x_{t \eta}^{(p+1)}\right)^{\text {net }}} \frac{\partial\left(x_{t \eta}^{(p+1)}\right)^{\text {net }}}{\partial \overline{x_{t b}^{(p)}}}\right] \sum_{\alpha=1}^{K_{p+2}} E_{t \alpha}^{(p+2)} \overline{w_{\alpha \eta}^{(p+1)}} \\
& =g_{p+1}^{\prime}\left(\overline{\left(x_{t \eta}^{(p+1)}\right)^{\mathrm{net}}}\right) \sum_{\alpha=1}^{K_{p+2}} \sum_{\beta=1}^{K_{p+1}} \frac{\partial E_{t \alpha}^{(p+2)}}{\partial \overline{x_{t \beta}^{(p+1)}}}\left[\frac{\partial \overline{x_{t \beta}^{(p+1)}}}{\partial\left(x_{t \beta}^{(p+1)}\right)^{\mathrm{net}}} \frac{\partial\left(x_{t \beta}^{(p+1)}\right)^{\text {net }}}{\partial \overline{x_{t b}^{(p)}}}\right. \\
& \left.+\frac{\partial \overline{x_{t \beta}^{(p+1)}}}{\partial \overline{\left(x_{t \beta}^{(p+1)}\right)^{\mathrm{net}}}} \frac{\partial \overline{\left(x_{t \beta}^{(p+1)}\right)^{\mathrm{net}}}}{\partial \overline{x_{t b}^{(p)}}}\right] \overline{w_{\alpha \eta}^{(p+1)}}
\end{aligned}
$$

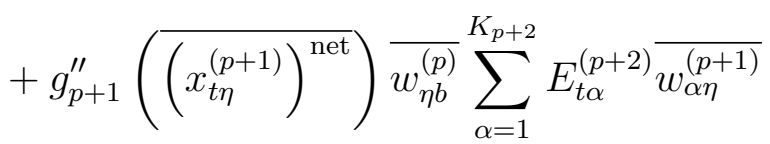

$$
\begin{aligned}
& =g_{p+1}^{\prime}\left(\overline{\left.\left(x_{t \eta}^{(p+1)}\right)^{\mathrm{net}}\right)} \sum_{\alpha=1}^{K_{p+2}} \sum_{\beta=1}^{K_{p+1}} \frac{\partial E_{t \alpha}^{(p+2)}}{\partial \overline{x_{t \beta}^{(p+1)}}}\right. \\
& \cdot g_{p+1}^{\prime}\left(\overline{\left.\left(x_{t \beta}^{(p+1)}\right)^{\text {net }}\right) \overline{w_{\beta b}^{(p)} w_{\alpha \eta}^{(p+1)}}}\right. \\
& +g_{p+1}^{\prime \prime}\left(\overline{\left(x_{t \eta}^{(p+1)}\right)^{\mathrm{net}}}\right) \overline{w_{\eta b}^{(p)}} \sum_{\alpha=1}^{K_{p+2}} E_{t \alpha}^{(p+2)} \overline{w_{\alpha \eta}^{(p+1)}} \\
& =\sum_{\beta=1}^{K_{p+1}}\left[\sum_{\alpha=1}^{K_{p+2}} \frac{\partial E_{t \alpha}^{(p+2)}}{\partial \overline{x_{t \beta}^{(p+1)}}} \overline{w_{\alpha \eta}^{(p+1)}}\right] \\
& \cdot g_{p+1}^{\prime}\left(\overline{\left(x_{t \eta}^{(p+1)}\right)^{\mathrm{net}}}\right) g_{p+1}^{\prime}\left(\overline{\left(x_{t \beta}^{(p+1)}\right)^{\mathrm{net}}} \overline{w_{\beta b}^{(p)}}\right. \\
& +\left[\sum_{\alpha=1}^{K_{p+2}} E_{t \alpha}^{(p+2)} \overline{w_{\alpha \eta}^{(p+1)}}\right] g_{p+1}^{\prime \prime}\left(\overline{\left(x_{t \eta}^{(p+1)}\right)^{\mathrm{net}}}\right) \overline{w_{\eta b}^{(p)}} .
\end{aligned}
$$




\section{Appendix B. Convergence of the One-Step Newton Steplength Algorithm of Real-Valued COMPLEX FunCTIONS}

Let $f: \Omega \subseteq \mathbb{C}^{k} \rightarrow \mathbb{R}$, and consider a general minimization algorithm with sequence of iterates $\{\mathbf{z}(n)\}$ given recursively by

$$
\mathbf{z}(n+1)=\mathbf{z}(n)-\mu(n) \mathbf{p}(n), n=0,1, \ldots,
$$

where $\mathbf{p}(n) \in \mathbb{C}^{k}$ such that $-\mathbf{p}(n)$ is the direction from the $n$th iterate to the $(n+1)$ th iterate and $\mu(n) \in \mathbb{R}$ is the learning rate or steplength which we allow to vary with each step. We are interested in guaranteeing that the minimization algorithm is a descent method, that is, that at each stage of the iteration the inequality $f(\mathbf{z}(n+1)) \leq f(\mathbf{z}(n))$ for $n=0,1, \ldots$ holds. Here, we provide details of the proof of the one-step Newton steplength algorithm for the minimization of real-valued functions on complex domains. Our treatment follows the exposition in [31], with the application to the complex Newton algorithm providing a proof of Theorem 6.1.

Lemma B.1. Suppose that $f: \Omega \subseteq \mathbb{C}^{k} \rightarrow \mathbb{R}$ is $\mathbb{R}$-differentiable at $\mathbf{z} \in \operatorname{int}(\Omega)$ and that there exists $\mathbf{p} \in \mathbb{C}^{k}$ such that $\operatorname{Re}\left(\frac{\partial f}{\partial \mathbf{z}}(\mathbf{z}) \mathbf{p}\right)>0$. Then there exists a $\delta>0$ such that $f(\mathbf{z}-\mu \mathbf{p})<f(\mathbf{z})$ for all $\mu \in(0, \delta)$.

Proof. Let $\mathbf{z}=\mathbf{x}+i \mathbf{y} \in \operatorname{int}(\Omega)$ with $\mathbf{x}, \mathbf{y} \in \mathbb{R}^{k}$. The function $f: \Omega \subseteq$ $\mathbb{C}^{k} \rightarrow \mathbb{R}$ is $\mathbb{R}$-differentiable at $\mathbf{z}$ if and only if $f: D \subseteq \mathbb{R}^{2 k} \rightarrow \mathbb{R}$ is (Frechet) differentiable at $(\mathbf{x}, \mathbf{y})^{T} \in \operatorname{int}(D)$, where $D$ is defined as in (??) and the (Frechet) derivative (equal to the Gateau derivative) at $(\mathbf{x}, \mathbf{y})^{T}$ is given by $\left(\frac{\partial f}{\partial \mathbf{x}}, \frac{\partial f}{\partial \mathbf{y}}\right)$. Suppose there exists $\mathbf{p}=\mathbf{p}_{R}+i \mathbf{p}_{I} \in$ $\mathbb{C}^{k}$ with $\mathbf{p}_{R}, \mathbf{p}_{I} \in \mathbb{R}^{k}$ such that $\operatorname{Re}\left(\frac{\partial f}{\partial \mathbf{z}}(\mathbf{z}) \mathbf{p}\right)>0$. Then using the coordinate and cogradient transformations (??) and (??) and the fact that $f$ is real-valued, we have the following ([30], pg. 34):

$$
\begin{aligned}
& \left(\frac{\partial f}{\partial \mathbf{x}}(\mathbf{x}, \mathbf{y}), \frac{\partial f}{\partial \mathbf{y}}(\mathbf{x}, \mathbf{y})\right)\left(\begin{array}{c}
\mathbf{p}_{R} \\
\mathbf{p}_{I}
\end{array}\right)=\left(\frac{\partial f}{\partial \mathbf{z}}(\mathbf{z}, \overline{\mathbf{z}}), \frac{\partial f}{\partial \overline{\mathbf{z}}}(\mathbf{z}, \overline{\mathbf{z}})\right) J \cdot \frac{1}{2} J^{*}\left(\begin{array}{c}
\mathbf{p} \\
\overline{\mathbf{p}}
\end{array}\right) \\
& =\frac{\partial f}{\partial \mathbf{z}}(\mathbf{z}, \overline{\mathbf{z}}) \mathbf{p}+\frac{\partial f}{\partial \overline{\mathbf{z}}}(\mathbf{z}, \overline{\mathbf{z}}) \overline{\mathbf{p}}=\frac{\partial f}{\partial \mathbf{z}}(\mathbf{z}) \mathbf{p}+\frac{\partial f}{\partial \mathbf{z}}(\mathbf{z}) \mathbf{p}=2 \operatorname{Re}\left(\frac{\partial f}{\partial \mathbf{z}}(\mathbf{z}) \mathbf{p}\right)>0 .
\end{aligned}
$$

By (8.2.1) in [31] there exists a $\delta>0$ such that $f\left((\mathbf{x}, \mathbf{y})-\mu\left(\mathbf{p}_{R}, \mathbf{p}_{I}\right)\right)<$ $f(\mathbf{x}, \mathbf{y})$ for all $\mu \in(0, \delta)$. Viewing $f$ again as a function on the complex domain $\Omega$, this is equivalent to the statement that $f(\mathbf{z}-\mu \mathbf{p})<$ $f(\mathbf{z})$ for all $\mu \in(0, \delta)$. 
Recall from Section VI that a stationary point of $f$ to be a stationary point in the sense of the function $f(\mathbf{z})=f(\mathbf{x}, \mathbf{y}): D \subseteq \mathbb{R}^{2 k} \rightarrow \mathbb{R}$. If $\hat{\mathbf{z}}=\hat{\mathbf{x}}+i \hat{\mathbf{y}}$ with $\hat{\mathbf{x}}, \hat{\mathbf{y}} \in \mathbb{R}^{k}$, then $\hat{\mathbf{z}}$ is a stationary point of $f$ if and only if $\frac{\partial f}{\partial \mathbf{x}}(\hat{\mathbf{x}}, \hat{\mathbf{y}})=\frac{\partial f}{\partial \mathbf{y}}(\hat{\mathbf{x}}, \hat{\mathbf{y}})=0$. Note that if $\operatorname{Re}\left(\frac{\partial f}{\partial \mathbf{z}}(\mathbf{z})\right) \neq 0$ for $\mathbf{z} \in \operatorname{int}(\Omega)$ (i.e. $\mathbf{z}$ is not a stationary point), then there always exists a $\mathbf{p} \in \mathbb{C}^{k}$ such that $\operatorname{Re}\left(\frac{\partial f}{\partial \mathbf{z}}(\mathbf{z}) \mathbf{p}\right)>0$. So this result is always true in the real domain, and the proof of Lemma B.1 only translates the result from the real domain to the complex domain.

For the sequence of iterates $\{\mathbf{z}(n)\}$ given by (B.1), we can find a sequence $\{\mathbf{p}(n)\}$ such that $\operatorname{Re}\left(\frac{\partial f}{\partial \mathbf{z}}(\mathbf{z}(n)) \mathbf{p}(n)\right)>0$ for $n=0,1, \ldots$ By Lemma B.1, for each $n$ there is at least one $\mu(n) \in(0, \infty)$ such that $f(\mathbf{z}(n)-\mu(n) \mathbf{p}(n))<f(\mathbf{z}(n))$. At each step in the algorithm we would like to make the largest descent in the value of $f$ as possible, so finding a desirable steplength $\mu(n)$ to guarantee descent translates into the real one-dimensional problem of minimizing $f(\mathbf{z}(n)-\mu \mathbf{p}(n))$ as a function of $\mu$. For each $n$ let $\mathbf{z}(n)=\mathbf{x}(n)+i \mathbf{y}(n)$ and $\mathbf{p}(n)=\mathbf{p}_{R}(n)+i \mathbf{p}_{I}(n)$ with $\mathbf{x}(n), \mathbf{y}(n), \mathbf{p}_{R}(n), \mathbf{p}_{I}(n) \in \mathbb{R}^{k}$ and write

$$
f(\mathbf{z}(n)-\mu \mathbf{p}(n))=f\left((\mathbf{x}(n), \mathbf{y}(n))-\mu\left(\mathbf{p}_{R}(n), \mathbf{p}_{I}(n)\right)\right) .
$$

Suppose $f$ is twice $\mathbb{R}$-differentiable on $\Omega$. As an approximate solution to this one-dimensional minimization problem we take $\mu(n)$ to be the minimizer of the second-degree Taylor polynomial (in $\mu$ )

$$
\begin{aligned}
T_{2}(\mu)=f( & \mathbf{x}(n), \mathbf{y}(n)) \\
& -\mu\left(\frac{\partial f}{\partial \mathbf{x}}(\mathbf{x}(n), \mathbf{y}(n)), \frac{\partial f}{\partial \mathbf{y}}(\mathbf{x}(n), \mathbf{y}(n))\right)\left(\begin{array}{c}
\mathbf{p}_{R}(n) \\
\mathbf{p}_{I}(n)
\end{array}\right) \\
& +\frac{1}{2} \mu^{2}\left(\begin{array}{c}
\mathbf{p}_{R}(n) \\
\mathbf{p}_{I}(n)
\end{array}\right)^{T} \mathcal{H}_{\mathbf{r r}}(\mathbf{x}(n), \mathbf{y}(n))\left(\begin{array}{c}
\mathbf{p}_{R}(n) \\
\mathbf{p}_{I}(n)
\end{array}\right)
\end{aligned}
$$

where $\mathcal{H}_{\text {rr }}$ denotes the real Hessian matrix

$$
\mathcal{H}_{\mathbf{r r}}=\left(\frac{\partial}{\partial \mathbf{x}}, \frac{\partial}{\partial \mathbf{y}}\right)\left(\frac{\partial f}{\partial \mathbf{x}}, \frac{\partial f}{\partial \mathbf{y}}\right)^{T} .
$$

If

$$
\left(\begin{array}{c}
\mathbf{p}_{R}(n) \\
\mathbf{p}_{I}(n)
\end{array}\right)^{T} \mathcal{H}_{\mathbf{r r}}(\mathbf{x}(n), \mathbf{y}(n))\left(\begin{array}{c}
\mathbf{p}_{R}(n) \\
\mathbf{p}_{I}(n)
\end{array}\right)>0
$$

then $T_{2}$ has a minimum at

$$
\mu(n)=\frac{\left(\frac{\partial f}{\partial \mathbf{x}}(\mathbf{x}(n), \mathbf{y}(n)), \frac{\partial f}{\partial \mathbf{y}}(\mathbf{x}(n), \mathbf{y}(n))\right)\left(\begin{array}{c}
\mathbf{p}_{R}(n) \\
\mathbf{p}_{I}(n)
\end{array}\right)}{\left(\begin{array}{c}
\mathbf{p}_{R}(n) \\
\mathbf{p}_{I}(n)
\end{array}\right)^{T} \mathcal{H}_{\mathbf{r r}}(\mathbf{x}(n), \mathbf{y}(n))\left(\begin{array}{c}
\mathbf{p}_{R}(n) \\
\mathbf{p}_{I}(n)
\end{array}\right)}
$$


(Note this is equivalent to taking one step toward minimizing $f$ over $\mu$ via the real Newton algorithm.) Using a computation similar to (B.2) in the proof of Lemma B.1, the denominator of (B.4) translates back into complex coordinates as ([30], pg. 38):

$$
\begin{aligned}
& \left(\begin{array}{c}
\mathbf{p}_{R}(n) \\
\mathbf{p}_{I}(n)
\end{array}\right)^{T} \mathcal{H}_{\mathbf{r r}}(\mathbf{x}(n), \mathbf{y}(n))\left(\begin{array}{c}
\mathbf{p}_{R}(n) \\
\mathbf{p}_{I}(n)
\end{array}\right) \\
& \quad=2 \operatorname{Re}\left\{\mathbf{p}(n)^{*} \mathcal{H}_{\mathbf{z z}}(\mathbf{z}(n)) \mathbf{p}(n)+\mathbf{p}(n)^{*} \mathcal{H}_{\overline{\mathbf{z} z}}(\mathbf{z}(n)) \overline{\mathbf{p}(n)}\right\} .
\end{aligned}
$$

Combining (B.4) with (B.5) and (B.2), if

$$
\operatorname{Re}\left\{\mathbf{p}(n)^{*} \mathcal{H}_{\mathbf{z z}}(\mathbf{z}(n)) \mathbf{p}(n)+\mathbf{p}(n)^{*} \mathcal{H}_{\overline{\mathbf{z} z}}(\mathbf{z}(n)) \overline{\mathbf{p}(n)}\right\}>0
$$

we can take the approximate solution to the minimization problem to be

$$
\mu(n)=\frac{\operatorname{Re}\left\{\frac{\partial f}{\partial \mathbf{z}}(\mathbf{z}(n)) \mathbf{p}(n)\right\}}{\operatorname{Re}\left\{\mathbf{p}(n) * \mathcal{H}_{\mathbf{z z}}(\mathbf{z}(n)) \mathbf{p}(n)+\mathbf{p}(n) * \mathcal{H}_{\overline{\mathbf{z}} \mathbf{z}}(\mathbf{z}(n)) \overline{\mathbf{p}(n)}\right\}} .
$$

Notice that $(\mathrm{B} .6)$ is in fact both a necessary and sufficient condition to obtain an approximate solution using (B.3) to the one-dimensional minimization problem of $f(\mathbf{z}(n)-\mu \mathbf{p}(n))$ over $\mu$, for if

$$
\operatorname{Re}\left\{\mathbf{p}(n)^{*} \mathcal{H}_{\mathbf{z z}}(\mathbf{z}(n)) \mathbf{p}(n)+\mathbf{p}(n)^{*} \mathcal{H}_{\overline{\mathbf{z}} \mathbf{z}}(\mathbf{z}(n)) \overline{\mathbf{p}(n)}\right\}<0
$$

the Taylor polynomial (B.3) attains only a maximum.

Since defining the sequence of steplengths $\{\mu(n)\}$ by (B.7) is only an approximate method, to guarantee the descent of the iteration, we consider further modification of the steplengths. From Lemma B.1, it is clear that we can choose a sequence of underrelaxation factors $\{\omega(n)\}$ such that

$$
f(\mathbf{z}(n)-\omega(n) \mu(n) \mathbf{p}(n))<f(\mathbf{z}(n))
$$

which guarantees that the iteration

$$
\mathbf{z}(n+1)=\mathbf{z}(n)-\omega(n) \mu(n) \mathbf{p}(n), n=0,1, \ldots
$$

is a descent method. We describe a way to choose the sequence $\{\omega(n)\}$.

First, recall some notation from Section VI. Suppose $\Omega$ is open and let $\mathbf{z}(0) \in \Omega$. The level set of $\mathbf{z}(0)$ under $f$ on $\Omega$ is defined by (6.1), and $L_{\mathbb{C}^{k}}^{0}(f(\mathbf{z}(0)))$ is the path-connected component of $L_{\mathbb{C}^{k}}(f(\mathbf{z}(0))$ containing $\mathbf{z}(0)$. Let $\|\cdot\|_{\mathbb{C}^{k}}: \mathbb{C}^{k} \rightarrow \mathbb{R}$ denote the Euclidean norm on $\mathbb{C}^{k}$, with $\|\mathbf{z}\|_{\mathbb{C}^{k}}=\sqrt{\mathbf{z}^{*} \mathbf{z}}$.

Lemma B.2 (Complex Version of the One-Step Newton Steplength Algorithm). Let $f: \Omega \subseteq \mathbb{C}^{k} \rightarrow \mathbb{R}$ be twice-continuously $\mathbb{R}$-differentiable 
on the open set $\Omega$. Suppose $L_{\mathbb{C}^{k}}^{0}(f(\mathbf{z}(0)))$ is compact for $\mathbf{z}(0) \in \Omega$ and that

$$
\eta_{0} \mathbf{h}^{*} \mathbf{h} \leq \operatorname{Re}\left\{\mathbf{h}^{*} \mathcal{H}_{\mathbf{z z}}(\mathbf{z}) \mathbf{h}+\mathbf{h}^{*} \mathcal{H}_{\overline{\mathbf{z} z}}(\mathbf{z}) \overline{\mathbf{h}}\right\} \leq \eta_{1} \mathbf{h}^{*} \mathbf{h}
$$

for all $\mathbf{z} \in L_{\mathbb{C}^{k}}^{0}(f(\mathbf{z}(0)))$ and $\mathbf{h} \in \mathbb{C}^{k}$, where $0<\eta_{0} \leq \eta_{1}$. Fix $\epsilon \in(0,1]$. Define the sequence $\{\mathbf{z}(n)\}$ using B.8 with $\mathbf{p}(n) \neq 0$ satisfying

$$
\operatorname{Re}\left(\frac{\partial f}{\partial \mathbf{z}}(\mathbf{z}(n))(\mathbf{p}(n))\right) \geq 0
$$

$\mu(n)$ defined by (B.7), and

$$
0<\epsilon \leq \omega(n) \leq \frac{2}{\gamma(n)}-\epsilon
$$

where, setting $\mathbf{z}=\mathbf{z}(n)$ and $\mathbf{p}=\mathbf{p}(n)$,

$$
\gamma(n)=\sup \left\{\begin{array}{c}
\frac{\operatorname{Re}\left\{\mathbf{p}^{*} \mathcal{H}_{\mathbf{z z}}(\mathbf{z}-\mu \mathbf{p}) \mathbf{p}+\mathbf{p}^{*} \mathcal{H}_{\overline{\mathbf{z}} \mathbf{z}}(\mathbf{z}-\mu \mathbf{p}) \overline{\mathbf{p}}\right\}}{\operatorname{Re}\left\{\mathbf{p}^{*} \mathcal{H}_{\mathbf{z z}}(\mathbf{z}) \mathbf{p}+\mathbf{p}^{*} \mathcal{H}_{\overline{\mathbf{z}} \mathbf{z}}(\mathbf{z}) \overline{\mathbf{p}}\right\}} \mid \\
\mu>0, \quad f(\mathbf{z}-\nu \mathbf{p})<f(\mathbf{z}) \\
\text { for all } \nu \in(0, \mu]
\end{array}\right\} .
$$

Then $\{\mathbf{z}(n)\} \subseteq L_{\mathbb{C}^{k}}^{0}(f(\mathbf{z}(0)))$,

$$
\lim _{n \rightarrow \infty} \frac{\operatorname{Re}\left(\frac{\partial f}{\partial \mathbf{z}}(\mathbf{z}(n))(\mathbf{p}(n))\right)}{\|\mathbf{p}(n)\|_{\mathbb{C}^{k}}}=0
$$

and $\lim _{n \rightarrow \infty}(\mathbf{z}(n)-\mathbf{z}(n+1))=0$.

Proof. Let $f: \Omega \subseteq \mathbb{C}^{k} \rightarrow \mathbb{R}$ be twice-continuously $\mathbb{R}$-differentiable on the open set $\Omega$, and define $D$ as in (??). Then $D$ is open and $f(\mathbf{x}, \mathbf{y}): D \subseteq \mathbb{R}^{2 k} \rightarrow \mathbb{R}$ is twice-continuously differentiable on $D$. Let $\mathbf{z}(0)=\mathbf{x}(0)+i \mathbf{y}(0) \in \Omega$ with $\mathbf{x}(0), \mathbf{y}(0) \in \mathbb{R}^{k}$ and set

$$
L_{\mathbb{R}^{2 k}}^{0}\left(f(\mathbf{x}(0), \mathbf{y}(0))=\left\{\left(\begin{array}{c}
\mathbf{x} \\
\mathbf{y}
\end{array}\right) \in D \mid \begin{array}{c}
\mathbf{x}, \mathbf{y} \in \mathbb{R}^{k}, \\
\mathbf{z}=\mathbf{x}+i \mathbf{y} \in L_{\mathbb{C}^{k}}^{0}(f(\mathbf{z}(0)))
\end{array}\right\} .\right.
$$

It is clear that since $L_{\mathbb{C}^{k}}^{0}(f(\mathbf{z}(0)))$ is assumed to be compact, the real level set $L_{\mathbb{R}^{2 k}}^{0}(f(\mathbf{x}(0), \mathbf{y}(0))$ is also compact.

Next, observe that for $\mathbf{z}=\mathbf{x}+i \mathbf{y} \in \mathbb{C}^{k}$ with $\mathbf{x}, \mathbf{y} \in \mathbb{R}^{k}$, if $\|\cdot\|_{R^{2 k}}$ : $\mathbb{R}^{2 k} \rightarrow \mathbb{R}$ denotes the Euclidean norm on $\mathbb{R}^{2 k}$, then

$$
\|\mathbf{z}\|_{\mathbb{C}^{k}}^{2}=\mathbf{z}^{*} \mathbf{z}=\left\|\left(\begin{array}{l}
\mathbf{x} \\
\mathbf{y}
\end{array}\right)\right\|_{\mathbb{R}^{2 k}}^{2}
$$


Using this fact and (B.5) we see that for $\mathbf{z}=\mathbf{x}+i \mathbf{y} \in L_{\mathbb{C}^{k}}^{0}(f(\mathbf{z}(0)))$ and $\mathbf{h}=\mathbf{h}_{R}+i \mathbf{h}_{I} \in \mathbb{C}^{k}$ with $\mathbf{x}, \mathbf{y}, \mathbf{h}_{R}, \mathbf{h}_{I} \in \mathbb{R}^{k}$ the condition (B.9) is equivalent to

$$
\eta_{0}^{\prime}\left\|\left(\begin{array}{c}
\mathbf{h}_{R} \\
\mathbf{h}_{I}
\end{array}\right)\right\|_{\mathbb{R}^{2 k}}^{2} \leq\left(\begin{array}{c}
\mathbf{h}_{R} \\
\mathbf{h}_{I}
\end{array}\right)^{T} \mathcal{H}_{\mathbf{r r}}(\mathbf{x}, \mathbf{y})\left(\begin{array}{c}
\mathbf{h}_{R} \\
\mathbf{h}_{I}
\end{array}\right) \leq \eta_{1}^{\prime}\left\|\left(\begin{array}{c}
\mathbf{h}_{R} \\
\mathbf{h}_{I}
\end{array}\right)\right\|_{\mathbb{R}^{2 k}}^{2},
$$

where again $\mathcal{H}_{\mathbf{r r}}$ denotes the real Hessian matrix of $f(\mathbf{x}, \mathbf{y}): D \subseteq$ $\mathbb{R}^{2 k} \rightarrow \mathbb{R}$, and $0<\eta_{0}^{\prime}=\frac{\eta_{0}}{2} \leq \frac{\eta_{1}}{2}=\eta_{1}^{\prime}$.

We have already seen in the proof of Lemma B.1 (see the calculation (B.2)) that the condition (B.10) on the vectors $\mathbf{p}(n)=\mathbf{p}_{R}(n)+i \mathbf{p}_{I}(n)$ with $\mathbf{p}_{R}(n), \mathbf{p}_{I}(n) \in \mathbb{R}^{k}$ is equivalent to the real condition

$$
\left(\frac{\partial f}{\partial \mathbf{x}}(\mathbf{x}(n), \mathbf{y}(n)), \frac{\partial f}{\partial \mathbf{y}}(\mathbf{x}(n), \mathbf{y}(n))\right)\left(\begin{array}{c}
\mathbf{p}_{R}(n) \\
\mathbf{p}_{I}(n)
\end{array}\right) \geq 0 .
$$

We have also seen that our choice (B.7) for $\mu(n)$ is equal to (B.4).

Finally, for $\epsilon \in(0,1]$, using (B.5) again we have the real analogue of (B.12):

$$
\begin{aligned}
& \gamma(n)= \\
& \sup \left\{\frac{\left(\begin{array}{c}
\mathbf{p}_{R}(n) \\
\mathbf{p}_{I}(n)
\end{array}\right)^{T} \mathcal{H}_{\mathbf{r r}}\left((\mathbf{x}(n), \mathbf{y}(n))-\mu\left(\mathbf{p}_{R}(n), \mathbf{p}_{I}(n)\right)\right)\left(\begin{array}{c}
\mathbf{p}_{R}(n) \\
\mathbf{p}_{I}(n)
\end{array}\right)}{\left(\begin{array}{c}
\mathbf{p}_{R}(n) \\
\mathbf{p}_{I}(n)
\end{array}\right)^{T} \mathcal{H}_{\mathbf{r r}}(\mathbf{x}(n), \mathbf{y}(n))\left(\begin{array}{c}
\mathbf{p}_{R}(n) \\
\mathbf{p}_{I}(n)
\end{array}\right)} \mid\right. \\
& \left.\begin{array}{c}
\mu>0, f\left((\mathbf{x}(n), \mathbf{y}(n))-\nu\left(\mathbf{p}_{R}(n), \mathbf{p}_{I}(n)\right)\right)<f(\mathbf{x}(n), \mathbf{y}(n)) \\
\text { for all } \nu \in(0, \mu]
\end{array}\right\} .
\end{aligned}
$$

By (B.2),

$$
\frac{\left(\frac{\partial f}{\partial \mathbf{x}}(\mathbf{x}(n), \mathbf{y}(n)), \frac{\partial f}{\partial \mathbf{y}}(\mathbf{x}(n), \mathbf{y}(n))\right)\left(\begin{array}{c}
\mathbf{p}_{R}(n) \\
\mathbf{p}_{I}(n)
\end{array}\right)}{\left\|\left(\begin{array}{c}
\mathbf{p}_{R}(n) \\
\mathbf{p}_{I}(n)
\end{array}\right)\right\|_{\mathbb{R}^{2 k}}}=\frac{2 \operatorname{Re}\left(\frac{\partial f}{\partial \mathbf{z}}(\mathbf{z}(n)) \mathbf{p}(n)\right)}{\|\mathbf{p}(n)\|_{\mathbb{C}^{k}}},
$$

so applying (14.2.9) in [31], $\left\{(\mathbf{x}(n), \mathbf{y}(n))^{T}\right\} \subseteq L_{\mathbb{R}^{2 k}}^{0}(f(\mathbf{x}(0), \mathbf{y}(0)))$,

$$
\lim _{n \rightarrow \infty} \frac{\left(\frac{\partial f}{\partial \mathbf{x}}(\mathbf{x}(n), \mathbf{y}(n)), \frac{\partial f}{\partial \mathbf{y}}(\mathbf{x}(n), \mathbf{y}(n))\right)\left(\begin{array}{c}
\mathbf{p}_{R}(n) \\
\mathbf{p}_{I}(n)
\end{array}\right)}{\left\|\left(\begin{array}{c}
\mathbf{p}_{R}(n) \\
\mathbf{p}_{I}(n)
\end{array}\right)\right\|_{\mathbb{R}^{2 k}}}=0,
$$

and

$$
\lim _{n \rightarrow \infty}\left(\left(\begin{array}{l}
\mathbf{x}(n) \\
\mathbf{y}(n)
\end{array}\right)-\left(\begin{array}{l}
\mathbf{x}(n+1) \\
\mathbf{y}(n+1)
\end{array}\right)\right)=0
$$


Translating back to complex coordinates yields the desired conclusion.

Assume that there is a unique stationary point $\hat{\mathbf{z}}$ in $L_{\mathbb{C}^{k}}^{0}(f(\mathbf{z}(0))$. We desire to guarantee that the sequence of iterates $\{\mathbf{z}(n)\}$ converges to $\hat{\mathbf{z}}$. Before we give conditions for convergence of the complex version of the one-step Newton steplength algorithm, recall from Section VI that the R-factors of a sequence $\{\mathbf{z}(n)\} \subseteq \mathbb{C}^{k}$ that converges to $\hat{\mathbf{z}} \in \mathbb{C}^{k}$ are given by $(6.2$, and the sequence has at least an $\mathrm{R}$-linear rate of convergence if $R_{1}\{\mathbf{z}(n)\}<1$.

Lemma B.3 (Convergence of the Complex Version of the One-Step Newton Steplength Algorithm). Let $f: \Omega \subseteq \mathbb{C}^{k} \rightarrow \mathbb{R}$ be twice-continuously $\mathbb{R}$-differentiable on the open convex set $\Omega$ and assume that $L_{\mathbb{C} k}^{0}(f(\mathbf{z}(0))$ is compact for $\mathbf{z}(0) \in \Omega$. Assume the notation as in Lemma B.2. Suppose for all $z \in \Omega$,

$$
\operatorname{Re}\left\{\mathbf{h}^{*} \mathcal{H}_{\mathbf{z z}}(\mathbf{z}) \mathbf{h}+\mathbf{h}^{*} \mathcal{H}_{\overline{\mathbf{z} z}}(\mathbf{z}) \overline{\mathbf{h}}\right\}>0 \text { for all } \mathbf{h} \in \mathbb{C}^{k}
$$

and assume that the $\mathbf{p}(n)$ are nonzero vectors satisfying

$$
\operatorname{Re}\left(\frac{\partial f}{\partial \mathbf{z}}(\mathbf{z}(n)) \mathbf{p}(n)\right) \geq C\left\|\left(\frac{\partial f}{\partial \mathbf{z}}(\mathbf{z}(n))\right)^{T}\right\|_{\mathbb{C}^{k}}\|\mathbf{p}(n)\|_{\mathbb{C}^{k}}, n=0,1, \ldots
$$

for some fixed $C>0$. Assume $f$ has a unique stationary point $\hat{\mathbf{z}}$ in $L_{\mathbb{C}^{k}}^{0}\left(f(\mathbf{z}(0))\right.$. Then $\lim _{n \rightarrow \infty} \mathbf{z}(n)=\hat{\mathbf{z}}$, and the rate of convergence is at least $R$-linear.

Proof. As in the proof of Lemma B.2, given the assumptions of this lemma, $f: D \subseteq \mathbb{R}^{2 k} \rightarrow \mathbb{R}$ is twice-continuously (Frechet) differentiable on the open convex set $D$, and the set $L_{\mathbb{R}^{2 k}}^{0}(f(\mathbf{x}(0), \mathbf{y}(0))$ is compact for $\mathbf{z}(0)=\mathbf{x}(0)+i \mathbf{y}(0) \in \Omega$, where $\mathbf{x}(0), \mathbf{y}(0) \in \mathbb{R}^{k}$.

Using (B.5), for $\mathbf{z}=\mathbf{x}+i \mathbf{y} \in \Omega$ the condition (B.13) is equivalent to the condition

$$
\left(\begin{array}{c}
\mathbf{h}_{1} \\
\mathbf{h}_{2}
\end{array}\right)^{T} \mathcal{H}_{\mathbf{r r}}(\mathbf{x}, \mathbf{y})\left(\begin{array}{c}
\mathbf{h}_{1} \\
\mathbf{h}_{2}
\end{array}\right)>0 \text { for all }\left(\begin{array}{c}
\mathbf{h}_{1} \\
\mathbf{h}_{2}
\end{array}\right) \in \mathbb{R}^{2 k} \text { with } \mathbf{h}_{1}, \mathbf{h}_{2} \in \mathbb{R}^{k}
$$

Thus for all $(\mathbf{x}, \mathbf{y})^{T} \in D$, the real Hessian $\mathcal{H}_{\mathbf{r r}}(\mathbf{x}, \mathbf{y})$ of $f$ is positive definite.

Also as in the proof of Lemma B.2, the real versions of the definitions of $\mu(n)$ and $\omega(n)$ given by (B.7) and (B.11), respectively, satisfy the real one-step Newton steplength algorithm (14.2.9) in [31]. 
Finally, for $\mathbf{z}=\mathbf{x}+i \mathbf{y} \in \mathbb{C}^{k}$ with $\mathbf{x}, \mathbf{y} \in \mathbb{R}^{k}$, a simple calculation shows that

$$
2\left\|\left(\frac{\partial f}{\partial \mathbf{z}}(\mathbf{z})\right)^{T}\right\|_{\mathbb{C}^{k}}=\left\|\left(\frac{\partial f}{\partial \mathbf{x}}(\mathbf{x}, \mathbf{y}), \frac{\partial f}{\partial \mathbf{y}}(\mathbf{x}, \mathbf{y})\right)^{T}\right\|_{\mathbb{R}^{2 k}}
$$

so using the calculation $(\mathrm{B} .2)$ in the proof of Lemma B.1, the condition (B.14) for the nonzero vectors $\mathbf{p}(n)=\mathbf{p}_{R}(n)+i \mathbf{p}_{I}(n)$ with $\mathbf{p}_{R}(n), \mathbf{p}_{I}(n) \in \mathbb{R}^{k}$ is equivalent to the real condition

$$
\begin{aligned}
&\left(\frac{\partial f}{\partial \mathbf{x}}(\mathbf{x}, \mathbf{y}), \frac{\partial f}{\partial \mathbf{y}}(\mathbf{x}, \mathbf{y})\right)\left(\begin{array}{c}
\mathbf{p}_{R}(n) \\
\mathbf{p}_{I}(n)
\end{array}\right) \\
& \geq C\left\|\left(\frac{\partial f}{\partial \mathbf{x}}(\mathbf{x}, \mathbf{y}), \frac{\partial f}{\partial \mathbf{y}}(\mathbf{x}, \mathbf{y})\right)^{T}\right\|_{\mathbb{R}^{2 k}}\left\|\left(\begin{array}{c}
\mathbf{p}_{R}(n) \\
\mathbf{p}_{I}(n)
\end{array}\right)\right\|_{\mathbb{R}^{2 k}}
\end{aligned}
$$

Thus we may apply Theorem (14.3.6) in [31] and transfer back to complex coordinates to obtain that $\lim _{n \rightarrow \infty} \mathbf{z}(n)=\hat{\mathbf{z}}$, where $\hat{\mathbf{z}}=\hat{\mathbf{x}}+i \hat{\mathbf{y}}$ with $\hat{\mathbf{x}}, \hat{\mathbf{y}} \in \mathbb{R}^{k}$ is the unique stationary point of $f$ in $L_{\mathbb{C}^{k}}^{0}(f(\mathbf{z}(0)))$, and the rate of convergence is at least $\mathrm{R}$-linear.

We now apply the previous results to the complex Newton algorithm. Let $f: \Omega \subseteq \mathbb{C}^{k} \rightarrow \mathbb{R}$ be twice-continuously $\mathbb{R}$-differentiable on the open convex set $\Omega$. Let $\mathbf{z}(0) \in \Omega$ and assume that the level set $L_{\mathbb{C}^{k}}^{0}(f(\mathbf{z}(0)))$ is compact. Suppose for all $\mathbf{z} \in \Omega$,

$$
\operatorname{Re}\left\{\mathbf{h}^{*} \mathcal{H}_{\mathbf{z z}}(\mathbf{z}) \mathbf{h}+\mathbf{h}^{*} \mathcal{H}_{\overline{\mathbf{z}} \mathbf{z}}(\mathbf{z}) \overline{\mathbf{h}}\right\}>0 \text { for all } \mathbf{h} \in \mathbb{C}^{k}
$$

As in the proof of Lemma B.3, this condition is equivalent to the positive definiteness of the real Hessian matrix $\mathcal{H}_{\mathbf{r r}}(\mathbf{x}, \mathbf{y})$ of $f$ for all $(\mathbf{x}, \mathbf{y})^{T} \in D$. Since $f$ is twice-continuously $\mathbb{R}$-differentiable, the Hessian operator $\mathcal{H}_{\mathbf{r r}}(\cdot): D \subseteq \mathbb{R}^{2 k} \rightarrow L\left(\mathbb{R}^{2 k}\right.$ ) (where $L\left(\mathbb{R}^{2 k}\right.$ ) denotes the set of linear operators $\left.\mathbb{R}^{2 k} \rightarrow \mathbb{R}^{2 k}\right)$ is continuous. Restricting to the compact set $L_{\mathbb{R}^{2 k}}^{0}(f(\mathbf{x}(0), \mathbf{y}(0)))$ we have that $\mathcal{H}_{\mathbf{r r}}(\cdot): L_{\mathbb{R}^{2 k}}^{0}(f(\mathbf{x}(0), \mathbf{y}(0))) \rightarrow$ $L\left(\mathbb{R}^{2 k}\right)$ is a continuous mapping such that $\mathcal{H}_{\mathbf{r r}}(\mathbf{x}, \mathbf{y})$ is positive definite for each vector $(\mathbf{x}, \mathbf{y})^{T} \in L_{\mathbb{R}^{2 k}}^{0}(f(\mathbf{x}(0), \mathbf{y}(0)))$. For each $(\mathbf{x}, \mathbf{y})^{T} \in$ $L_{\mathbb{R}^{2 k}}^{0}(f(\mathbf{x}(0), \mathbf{y}(0)))$ set

$$
\tilde{\mathbf{p}}(\mathbf{x}, \mathbf{y})=\mathcal{H}_{\mathbf{r r}}(\mathbf{x}, \mathbf{y})^{-1}\left(\frac{\partial f}{\partial \mathbf{x}}(\mathbf{x}, \mathbf{y}), \frac{\partial f}{\partial \mathbf{y}}(\mathbf{x}, \mathbf{y})\right)^{T}
$$


By Lemma (14.4.1) in [31, there exists a constant $C>0$ such that

$$
\begin{aligned}
\left(\frac{\partial f}{\partial \mathbf{x}}(\mathbf{x}, \mathbf{y}),\right. & \left.\frac{\partial f}{\partial \mathbf{y}}(\mathbf{x}, \mathbf{y})\right) \tilde{\mathbf{p}}(\mathbf{x}, \mathbf{y}) \\
& \geq C\left\|\left(\frac{\partial f}{\partial \mathbf{x}}(\mathbf{x}, \mathbf{y}), \frac{\partial f}{\partial \mathbf{y}}(\mathbf{x}, \mathbf{y})\right)^{T}\right\|_{\mathbb{R}^{2 k}}\|\tilde{\mathbf{p}}(\mathbf{x}, \mathbf{y})\|_{\mathbb{R}^{2 k}}
\end{aligned}
$$

for all $(\mathbf{x}, \mathbf{y})^{T} \in L_{\mathbb{R}^{2 k}}^{0}(f(\mathbf{x}(0), \mathbf{y}(0)))$. As in the proof of Lemma B.3. B.15 is equivalent to the inequality

$$
\operatorname{Re}\left(\frac{\partial f}{\partial \mathbf{z}}(\mathbf{z}) \mathbf{p}(\mathbf{z})\right) \geq C\left\|\left(\frac{\partial f}{\partial \mathbf{z}}(\mathbf{z})\right)^{T}\right\|_{\mathbb{C}^{k}}\|\mathbf{p}(\mathbf{z})\|_{\mathbb{C}^{k}}
$$

for all $\mathbf{z} \in L_{\mathbb{R}^{2 k}}^{0}(f(\mathbf{x}(0), \mathbf{y}(0)))$, where

$$
\begin{aligned}
& \tilde{\mathbf{p}}(\mathbf{z})=- {\left[\mathcal{H}_{\mathbf{z z}}(\mathbf{z})-\mathcal{H}_{\overline{\mathbf{z}} \mathbf{z}}(\mathbf{z}) \mathcal{H}_{\overline{\mathbf{z}} \mathbf{z}}(\mathbf{z})^{-1} \mathcal{H}_{\mathbf{z} \overline{\mathbf{z}}}(\mathbf{z})\right]^{-1} } \\
& \cdot\left[\mathcal{H}_{\overline{\mathbf{z}} \mathbf{z}}(\mathbf{z}) \mathcal{H}_{\overline{\mathbf{z}}}(\mathbf{z})^{-1}\left(\frac{\partial f}{\partial \overline{\mathbf{z}}}(\mathbf{z})\right)^{*}-\left(\frac{\partial f}{\partial \mathbf{z}}(\mathbf{z})\right)^{*}\right]
\end{aligned}
$$

is obtained from $\tilde{\mathbf{p}}(\mathbf{x}, \mathbf{y})$ (where $\mathbf{z}=\mathbf{x}+i \mathbf{y}$ ) using the coordinate and cogradient transformations (??) and (??), respectively [30]. Suppose $f$ has a unique stationary point $\hat{\mathbf{z}}$ in $L_{\mathbb{C}^{k}}^{0}(f(\mathbf{z}(0)))$. Consider the iteration

$$
\mathbf{z}(n+1)=\mathbf{z}(n)-\omega(n) \mu(n) \mathbf{p}(n), n=0,1, \ldots,
$$

where the $\mathbf{p}(n)$ are the nonzero complex Newton updates defined by $\mathbf{p}(n)=\tilde{\mathbf{p}}(\mathbf{z}(n))$, and assume the notation of Lemma B.2. Then $\{\mathbf{z}(n)\} \subseteq$ $L_{\mathbb{C}^{k}}^{0}(f(\mathbf{z}(0)))$. The vectors $\mathbf{p}(n)$ satisfy (B.14), so by Lemma B.3 the sequence of iterates $\{\mathbf{z}(n)\}$ converges to $\mathbf{z}$, and the rate of convergence is at least R-linear. Thus we have proved Theorem 6.1. 